\title{
Lab-based operando x-ray photoelectron spectroscopy for probing low-volatile liquids and their interfaces across a variety of electrosystems
}

Cite as: J. Vac. Sci. Technol. A 38, 040805 (2020); doi: 10.111 16/6.0000273

Submitted: 20 April 2020 . Accepted: 10 June 2020 .

Published Online: 29 June 2020

Pinar Aydogan Gokturk, (D) Merve Taner Camci, (D) and Sefik Suzer ${ }^{a)}$ (D)

\section{AFFILIATIONS}

Department of Chemistry, Bilkent University, 06800 Ankara, Turkey

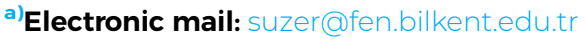

\begin{abstract}
The understanding of fundamental processes in liquids and at the liquid/electrode interfaces of electrochemical systems is crucial for the development of new devices and technologies with higher efficiency and improved performance. However, it is generally difficult to isolate and study the component of interest in such complex systems. Additionally, ex situ analyses do not always reflect the same properties under operating conditions. Hence, operando characterization tools are required for observing related electrical and chemical processes directly at the places where and while they occur. Operando x-ray photoelectron spectroscopy (o-XPS) has been used, while the sample is imposed to $\mathrm{DC} / \mathrm{AC}$ voltage stress, to record the binding energy shifts in and on liquids and their interfaces to extract local potentials, as well as many related properties specific to the application in a noncontact and chemically resolved fashion. The applications of o-XPS to low-volatile liquids shown in this review span well-defined studies of (1) electrochemical cells, (2) double-layer capacitors, and (3) electrowetting on dielectrics. The methodology and several applications selected from the authors' recent publications are presented.
\end{abstract}

\section{Published under license by AVS. https://doi.org/10.1116/6.0000273}

\section{INTRODUCTION}

XPS (x-ray photoelectron spectroscopy), originally referred as electron spectroscopy for chemical analysis (ESCA), is a surface sensitive technique, which has long been utilized to shed light on chemical, physical, or structural changes following certain processes. ${ }^{1}$ With the increasing amount of accessibility of these instruments, XPS has become a well-known routine to study surfaces and interfaces of mostly solid samples. However, a facile advantage of XPS to carry information on local potential is relatively unknown and not appreciated enough. These surface science investigations are traditionally performed either as an ex situ one, by comparing the surface composition before and after a specific process is applied, ${ }^{2-4}$ or in operando, by interrogating the surface of the sample while under processes like exposing them to chemical, ${ }^{5}$ electrical, ${ }^{6}$ or photochemical stimuli. ${ }^{7}$ Under electrical excitation, the core level photoelectrons measured by XPS show a shift in kinetic energy $(\Delta \mathrm{KE})$ that is related to the local potential $(\mathrm{V})$ on the surface by $\Delta$
$(\mathrm{KE})=\mathrm{eV}$ and is experimentally recorded as an apparent binding energy (ABE) shift $\triangle(A B E){ }^{8}$ This review will mainly focus on XPS analyses of samples and devices while imposing electrical stimuli only, since it is the most common form utilized for analyses of new materials in electrochemical and energy related fields.

Operando XPS (o-XPS), utilizing the application of electrical voltage bias [in either the direct current (DC) or alternating current (AC) forms] during data acquisition, is powerful due to its ability to provide electrical, in addition to the traditional chemical, information about the sample. This technique, especially under AC actuation, has been developed and extensively used by our group. ${ }^{9-11}$ While DC excitation during data acquisition provides steady state information, AC excitation helps to understand the dynamics of electrical potential developments. Real time observation of fast processes occurring in a time of a few milliseconds is difficult to observe by conventional XPS studies under laboratory conditions. However, varying the frequency of the AC excitation helps to catch processes in action. For such analysis, time-resolved 
$\mathrm{x}$-ray photoelectron spectroscopy with a fast data gathering snapshot mode, where spectra are recorded continuously as a function of time while exposing the surface to some type of external stimuli, is used to follow the temporal evolution of electrical potential on the surface on the milliseconds to hundreds of seconds time scales.

Even though XPS measurements on solids has become a wellknown routine, and the investigation of liquids dates back about five decades, ${ }^{12-14}$ extensive use of it for liquids has been relatively slow, since it is challenging to implement this traditionally ultrahigh vacuum (UHV) technique to liquids. The study of liquids or solid/liquid interfaces requires new technical designs to overcome obstacles arising from the following: (i) most liquids are showing high vapor pressures, not compatible with UHV, and (ii) electrons emitted from liquids undergo multiple collisions with both the liquid and vapor molecules until reaching the detector and results in significant loss of the signal. Although ambient pressure XPS allow studies at elevated pressures by modifying the apparatus to decrease the distance that the electron travels and applying differential pumping stages, analysis of liquids or solid/liquid interfaces still requires sophisticated and complex instrumentation. Only special methods, such as the dip and pull method, ${ }^{15,16}$ liquid micro-jet, ${ }^{17}$ and/or liquid cell, ${ }^{18}$ where a membrane of choice separates the liquid from the high vacuum chamber, can be used for solid/liquid interface analysis. Ambient pressure XPS systems are nowadays available as established and long-utilized synchrotron facilities, as well as lab-based instruments. ${ }^{13,19-24}$ Historical developments of the latter has recently been presented. ${ }^{25}$

In the past decade, a different investigation route was pursued utilizing mostly lab-based instruments and using low-volatile liquids that usually have sufficient electrical conductivity, mainly, ionic liquids (ILs) and/or polymeric electrolytes. This line of research has paved the way to probe in situ electrochemical properties and/or reactions with chemical specificity, rendering a wealth of novel electrochemical information. ${ }^{26-32}$

Using a lab-based XPS instrument in operando, we have also started investigating ionic liquids and low-volatile organic compounds as well as a number of different devices fabricated containing them as the electrolyte or the electrochemical reaction media about five years ago. Major findings of these investigations have already been published. Our first work described the investigation of a coplanar capacitor for harvesting information from binding energy shifts of the F1s core level representing the ionic liquid, following polarization of one of the metal electrodes by application of external DC or AC electrical bias. ${ }^{33} \mathrm{We}$, then, extended it to probing the nature of an electrochemical corrosion process between the ionic liquid and Au electrodes, ${ }^{34}$ and finally to detect, in situ, an electrochemically generated carbene formation ${ }^{35}$ and its $\mathrm{CS}_{2}$ adduct. $^{36}$ Most recently, we have utilized o-XPS to probe electrowetting properties of ionic and other low-volatile liquids under both DC, ${ }^{37}$ and AC (Ref. 38) actuations, in order to extract chemically resolved electrical information of such systems, guided by modeling. ${ }^{39,40}$ ILs and other low-volatile liquids have witnessed a colossal research activity due, mostly, to their potential use in battery and other energy harvesting and/or storage applications. ${ }^{41}$

Within the scope of the present contribution, we will review exemplary cases of o-XPS experiments performed by our group, emphasizing mostly the methodology, as well as pointing out the new findings and developments to illustrate that XPS can be used as a contactless probe of local electrical potential. We have organized this material into two different classes of electrical excitation: DC and AC actuations and their applications. We begin with discussions of XPS experiments on liquid surfaces and then extend our understanding to solid/liquid interfaces. These examples, for the application of o-XPS on electrosystems presented, address the following questions: (1) How to follow electrochemical reactions and electrowetting processes; (2) How 2D area detection during XP data acquisition can be used to visualize local potentials across devices and generate equipotential lines; and (3) How varying the frequency of $\mathrm{AC}$ excitation helps to catch the otherwise invisible temporal evolution of the potentials on the surface on milliseconds to seconds time scales, and at different and relatively large, micro- to millimeters, lateral locations.

\section{EXPERIMENT}

The ionic liquids used, 1-ethyl-3-methylimidazolium acetate (EMIm-Ac), 1-buthyl-3-methylimidazolium hexafluoro-phosphate (BMIm-PF $)_{6}$, and $N, N$-diethyl- $N$-methyl- $N$-(2-methoxyethyl) ammonium bis (trifluoromethanesulfonyl) imide (DEME-TFSI), were purchased from Sigma-Aldrich, and the fourth one, $\mathrm{N}$ - $\mathrm{N}$-diethyl$\mathrm{N}$-methyl- $\mathrm{N}$-(2-metoxyethyl)-ammonium tetra-fluroborate (DEME- $\left.\mathrm{BF}_{4}\right)$ was purchased from Io-Li-Tec. Liquid polyethylene glycol (PEG) with $600 \mathrm{amu}$ was also purchased from Sigma-Aldrich. In our investigations, the liquids were used without any purification and either in the form of a small ( $\sim 2 \mathrm{~mm}$ in diameter) sessile drop on a suitable substrate or as a thin electrolyte layer between two coplanar electrodes. For investigation of sessile drops on $\mathrm{Si}$ wafers or $\mathrm{SiO}_{2} / \mathrm{Si}$ dielectrics coated with a thin hydrophobic layer of either CYTOP or Teflon-1600, a thin gold wire was inserted into droplet for bias applications. For fabricating devices in the coplanar form, two gold metal electrodes were evaporated onto a $20 \mu \mathrm{m}$ thick porous polyethylene membrane (PEM) (Celgard 2730, Gelon LIB Group) with porosity of $43 \%$ and the liquids were introduced by soaking them to the membrane in between the two electrodes in the source-drain geometry. Figure 1 depicts different geometries used for the various applications to be presented. Note that, although the substrate is shown as the grounded electrode, the bias can also be introduced through the substrate, which will be shown to be crucial especially for AC actuations.

A Thermo Fisher K-Alpha X-ray Photoelectron Spectrometer with monochromatized $\mathrm{x}$-rays of $1486.6 \mathrm{eV}$ was used to collect all data for the XPS measurements. Spectrometer pass energies of $200 \mathrm{eV}$ for the survey spectra and $50 \mathrm{eV}$ for high-resolution spectra of all elemental spectral regions were used. For line scan and areal measurements, x-ray spot sizes of either 50 or $100 \mu \mathrm{m}$ were used. For the time-resolved XPS, fast data gathering, the snapshot mode was used, with a time resolution of $1 \mathrm{~s}$, instead of the scanning mode. Pressure in the analyzing chamber was always kept under $10^{-8}$ mbar. The external DC bias was applied using a Keithley 2400 Source Meter. For pulsed and the frequency dependent AC measurements, a Stanford Research System Model DS340 Function Generator was used. All data fittings were carried out by the AVANTAGE software equipped with the instrument using a symmetrical 


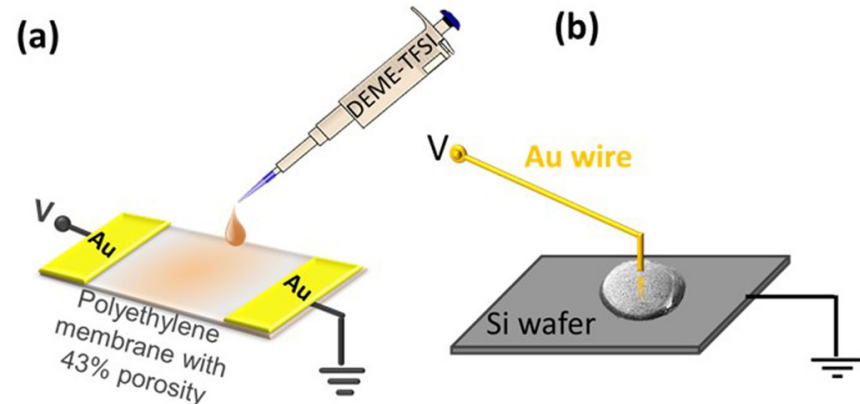

Gaussian/Lorentzian product function with 30/70 ratio after Shirley background subtraction.

\section{DC ACTUATION AND ITS APPLICATIONS}

Unlike optical spectroscopic techniques, XPS is a chargedparticle-based chemical analysis technique that is extremely sensitive to the electrical potentials developed due to uncompensated charges and/or external biases imposed. As a result, the measured binding energies undergo shifts, which are quite informative when recorded faithfully. For example, in Fig. 2, we show the C1s spectra of the PEG drop on a reliable dielectric substrate under application of various DC bias until the system breaks down.

Representative XP spectra of the grounded PEG liquid drop in a setup that is shown in Fig. 1(c) are depicted in Fig. 2. In addition to the main $\mathrm{C} 1 \mathrm{~s}$ and $\mathrm{O} 1 \mathrm{~s}$ peaks of the material, a second peak appears as a shoulder in the lower energy side of the C1s peak, which is curve-resolved and assigned to the adventitious carbon. (c)

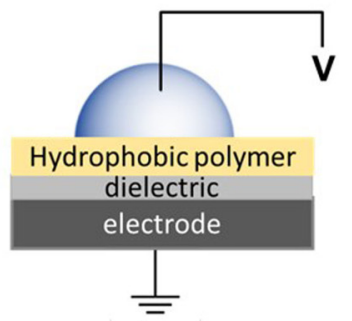

FIG. 1. Geometries used in this work (a) Liquid film introduced in between two deposited $\mathrm{Au}$ electrodes on an insulating porous membrane substrate. Liquid in the form of a sessile drop on (b) Si and (c) hydrophobic dielectric substrate.

With the increase of external bias applied from the wire, change in the apparent binding energy is shown in Figs. 2(b) and 2(c). Since no current passes through the system, the entire potential drops occur at the electrode/electrolyte interfaces. Additionally, no measurable charging is observable due to finite conductivity of the liquid, and only trivial shifts are observable everywhere on the drop surface, i.e., the magnitude of the measured binding energy shifts (in $\mathrm{eV}$ ) equal to the applied bias voltage (V), until the breakdown. Therefore, the slope in Fig. 2(c) is almost 1.

For the instrument we have used, we were able to faithfully record trivial shifts under DC biases as large as $500 \mathrm{~V}$ on strong and reliable dielectrics. However, more important is that, the detection of different and measurable nontrivial shift underpins the change(s) in electrical properties of the materials and/or devices under investigation, such as the occurrence of a dielectric breakdown [Fig. 2(b)], or others, as will be shown later.

On the other hand, with the other two device configurations we use, the liquid behaves like an electrolyte between two electrodes

(a)

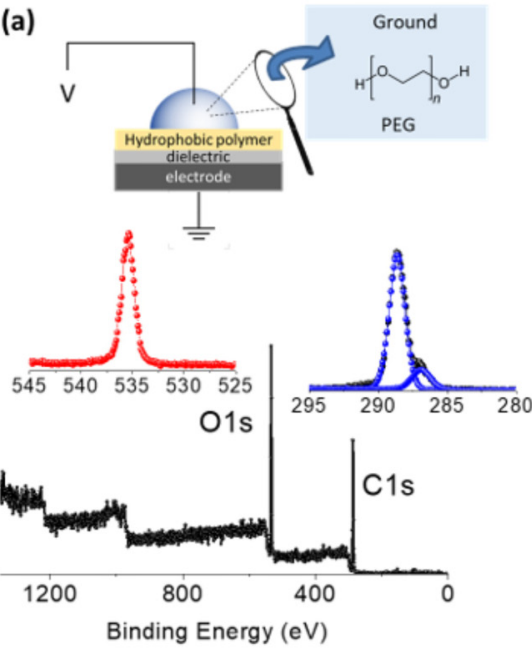

(b)

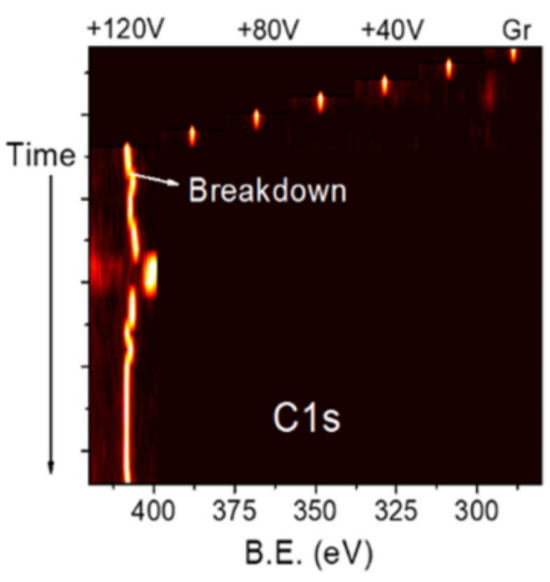

(c)

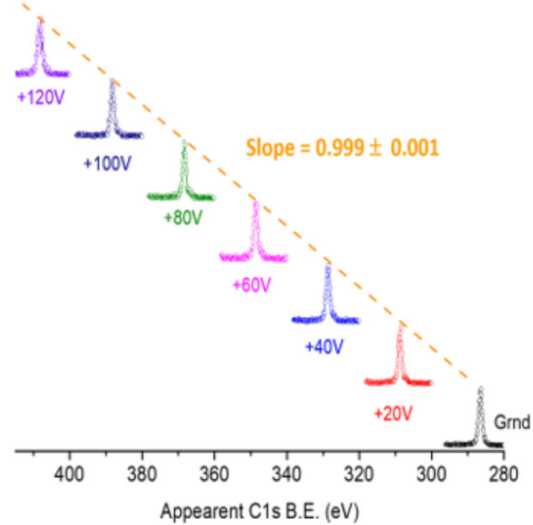

FIG. 2. (a) Schematics of the experimental setup and XP survey spectrum of the PEG droplet in the scanning mode with higher resolution of the C1s and O1s regions. (b) C1s spectra continuously recorded at different DC bias increased with $20 \mathrm{~V}$ steps until $+120 \mathrm{~V}$, and held at that bias, which triggers sporadic dielectric breakdown events. Note that few partial and/or full recovery (self-healing) processes are also observable. (c) C1s region under similar bias steps. Adapted with permission from Aydogan Gokturk et al., Langmuir 34, 7301 (2018). Copyright 2018, American Chemical Society. 
and allows the passage of current as in Figs. 1(a) and 1(b); therein, we expect that XPS core level peaks from the electrolyte elements to show certain $\mathrm{ABE}$ shifts, reflecting the electrical double layer (EDL) and corresponding potential drops at the interfaces. As a particular example, Fig. 3 shows the electrode Au4f and electrolyte F1s spectral features along the line from the source to the drain electrodes under +3 and $-3 \mathrm{~V}$. No shift is observed on Au4f spectra taken from drain electrode. This observation is consistent with the fact that the drain electrode is grounded with the XPS analyzer; hence, no electrical potential shift, i.e., ABE shift, is expected. Similarly, ABE shifts of Au4f from the source electrode match exactly the applied potential. However, the F1s and also the other IL core levels exhibit constant, flat, and nonvarying ABE shifts across the electrodes. Moreover, these measured shifts correspond to only half of the imposed potential and the rest is partitioned equally between the two gold-IL interfaces, as depicted in Fig. 3(c).

Because our instrumental $\mathrm{x}$-ray spot size is much larger than the thickness of the EDLs at the two interfaces, it was not possible to follow the potential developments within the double layer. However, in the same year with a different experimental geometry, and using tender $\mathrm{x}$-rays, which excited photoelectrons to have larger inelastic mean free paths and, therefore, ability to probe deeper, Favaro et al. have succeeded in probing the shape of the EDL at the solid/liquid interface. ${ }^{24}$

\section{A. In situ electrochemical carbene generation in ionic liquids}

Carbene compounds have always been assumed to be too shortlived intermediates for any useful study and they easily undergo dimerization. The ground-breaking work by Arduengo et al., showing that "bottle-able" carbenes could be synthesized, resulting in a fundamental shift in this field. ${ }^{42}$ The molecule reported in the study (1,3-di-l-adamantylimidazol-2-ylidene) contains a fivemembered imidazole ring, which is substituted with bulky adamantyl groups. Following this, a large body of work has appeared reporting synthesis of mostly $\mathrm{N}$-heterocyclic carbenes (NHC). This field has been reviewed by Hopkinson et al. ${ }^{43}$

NHCs have most commonly been synthesized starting with a 1, 3-disubstituted imidazolium salts to generate the carbene via deprotonation using a strong base. ${ }^{44,45}$ Alternatively, it was also shown that chemical or electrochemical reduction of imidazolium salts under the right conditions leads to an NHC. ${ }^{46}$ Chemically or electrochemically generated NHCs have been shown to be excellent ligands for metal catalysts for a number of reactions due to their strong $\sigma$-donor properties. ${ }^{47}$

A different route was followed by us where XPS was utilized to follow an in situ electrochemical reduction of the imidazolium cation leading to the production of stable NHC under UHV conditions. Figure 4 shows XP spectra of the EMIm-Ac ionic liquid before applying any voltage bias. As is evident from the figure, the pristine sample shows only a single peak in the N1s region corresponding to the two equivalent nitrogen atoms of the imidazolium ring, carrying overall one unit of positive charge, at the binding energy of $402.2 \mathrm{eV}^{48-51}$

Contribution to $\mathrm{C} 1 \mathrm{~s}$ envelope comes both from the EMIm and the acetate ions. The specific carbons are numbered on the structure that is drawn to illustrate the various contributions. We have adopted the published binding energy values for the $\mathrm{C} 1 \mathrm{~s}$ peaks of the cation ${ }^{48}$ and the guidance outlined for others. ${ }^{26}$
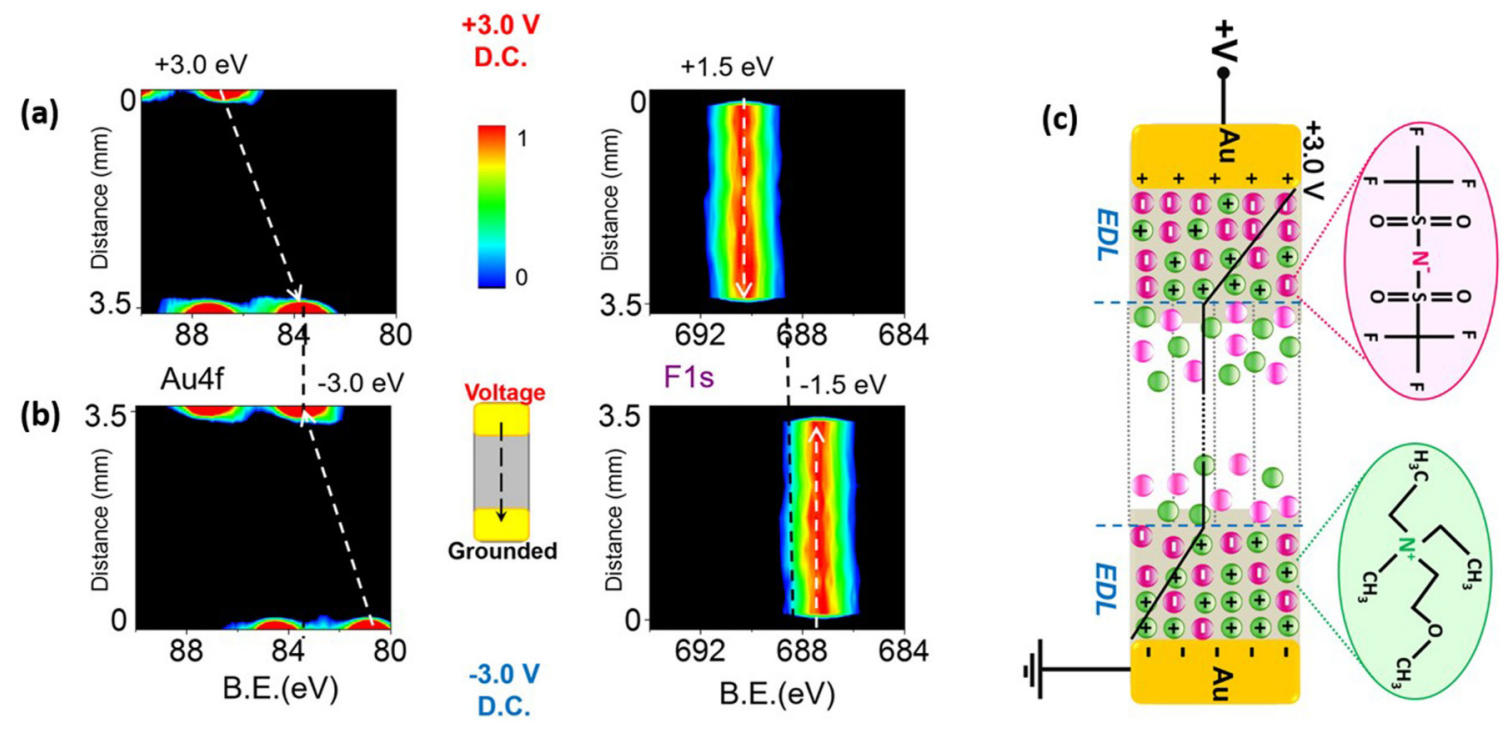

FIG. 3. XP spectra of Au4f and F1s regions, recorded in the line scan mode between the two gold electrodes, containing a porous polymer film impregnated with the ionic liquid DEME-TFSI, under the application of $(a)+3 V$ or $(b)-3 V D C$ bias. (c) Schematic representation of electrical potentials developed on the surface under $+3 \mathrm{~V} D C$ bias. The potential is screened at both interfaces by the two EDLs formed. Adapted with permission from Camci et al., Phys. Chem. Chem. Phys. 18, 28434 (2016). Copyright 2016, PCCP Owner Societies. 
1-Ethyl-3-Methyl Imidazolium Acetate

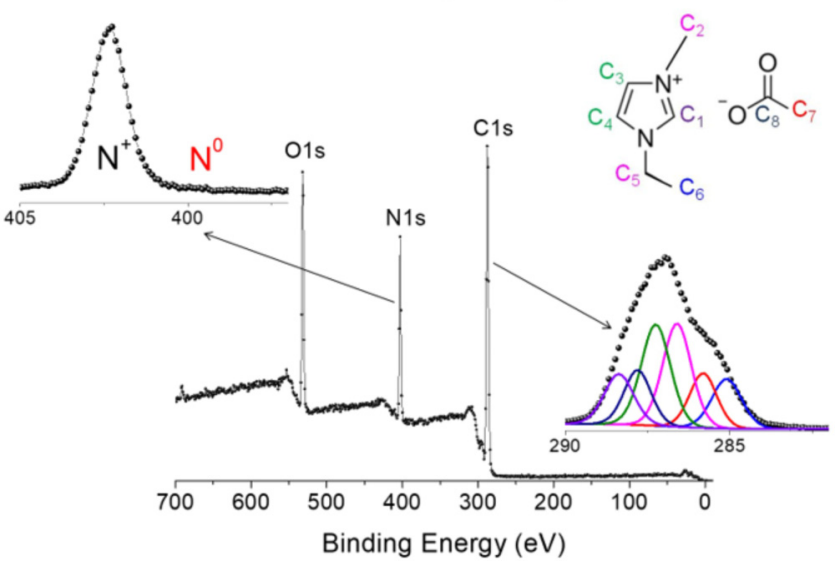

FIG. 4. Survey spectrum of the EMIm-Ac and the N1s and C1s levels recorded at a higher resolution. The N1s region consists of a single peak assignable to the two equivalent nitrogen atoms of the cyclic structure. The $\mathrm{C} 1 \mathrm{~s}$ region is complex and is fitted to eight peaks, two of which have been fitted to two equivalent carbons as indicated in the inset. Reprinted with permission from Aydogan Gokturk et al., Electrochim. Acta 234, 37 (2017). Copyright 2017, Elsevier.

Binding energy scale was calibrated by setting aliphatic carbon (C6) to $285.00 \mathrm{eV}^{52}$

With this device geometry, electroactive imidazolium species are not only the reactant but also part of the ionic liquid medium that acts as the electrolyte. Once proper bias is applied, a reduction is expected on the negatively polarized electrode that would lead to carbene formation. Indeed, within $7 \mathrm{~h}$, the carbene species can be seen to form close to the negatively polarized silicon wafer, as shown in Fig. 5. We observe a new N1s signal appearing at a binding energy that is $\sim 2.5 \mathrm{eV}$ lower than that of the N1s of the imidazolium ring, which is attributed to the two nitrogen atoms that are part of the overall neutral NHC.

When a positive bias is applied to the gold wire electrode, the silicon electrode is effectively at $-3 \mathrm{~V}$ against the gold wire, which enables the silicon electrode to reduce the imidazolium cation, leading to the formation of the carbene. When a negative bias is applied to the gold electrode, reduction happens on the gold electrode, and the carbene N1s signal becomes more visible in that vicinity. Both of these expectations are verified by results displayed in Fig. 5, in which the first appearance of the new peak is in the vicinity of the Si-electrode and moves within the liquid drop up toward the gold wire electrode in time, and only after switching the bias to $-3 \mathrm{~V}$, the intensity grows near that electrode. Note that the electrical potential within the electrolyte medium is not half of the applied potential as in the case of coplanar gold electrodes that we mentioned previously because of nonequal capacitance values of the two solid/liquid interfaces. In parallel, oxidized Si moieties ( $\mathrm{SiOx})$ are also produced within the IL medium (not shown). The interpretation of the results is supported by both observation of reversible redox peaks in the voltammogram (Fig. 6) and the density functional theory calculations. ${ }^{35}$
The cyclic voltammetry data inside the XPS chamber show that the substituted imidazolium cation species can get reduced to the related carbene species in a chemically reversible manner. This reversibility shows that, in the absence of any oxygen and water, the carbene is stable in vacuum within the time scales of the experiment.

This work demonstrated, for the first time, that reversible carbene electrochemistry can be followed inside an XPS chamber. The new N1s peak with the binding energy of $399.8 \mathrm{eV}$ generated via electrochemical means is assigned to the reduced $\mathrm{N}$ atoms of the NHC.

\section{B. Electrochemical generation of Au nanoparticles in an ionic liquid}

In addition to conventional chemical synthetic routes, metal nanoparticles (NPs) have also been prepared using metal ions and chemical reducers in IL media by various routes like photon or ion beam irradiation. ${ }^{53-60}$ It was reported that Au nano- and microstructures could be prepared and stabilized using dissolved Au salt without the need for additional reducing and stabilizing agents, but using only the IL for the reaction medium. ${ }^{61}$ As mentioned in the Introduction, we had earlier reported on an XPS study monitoring the potential screening across two gold electrodes fabricated on a porous polymer surface, which was impregnated with an IL (DEME-TFSI). ${ }^{33}$ In that work, we had also mentioned that the prolonged application of $+3 \mathrm{~V}$ bias led to the formation of Au NPs in the vicinity of the polarized electrode (i.e., oxidation-mediated electrocorrosion) but within the IL medium. In a later paper, we had followed that process and characterized the Au NPs produced, which will be summarized in this section. ${ }^{34}$ The two Au electrodes used in a coplanar capacitor geometry offered a convenient platform for elucidating the mechanism(s) of this electrocorrosion process, because of the symmetric nature of the electrodes in terms of their chemical makeup and size.

In Fig. 7 , we depict the Au4f region recorded under a $+3 \mathrm{~V}$ DC stress, while recording consecutive XP spectra in the line scan mode as snapshots, with $100 \mu \mathrm{m} \mathrm{x}$-ray spot and $100 \mu \mathrm{m}$ step sizes between each data point, starting from the source electrode toward the drain, as shown in Fig. 7. This voltage caused a current flow between the electrodes, which was high at the beginning but reached a steady state value of $\sim 14 \mathrm{nA}$ as measured by the source meter. This current also caused corrosion at the anodically polarized $\mathrm{Au}$ electrode. The corrosion was noticeable by the physical changes in the electrode accompanied by the formation of secondary $\mathrm{Au}$ species at the electrode/IL interfacial region, via examining the Au4f spectra recorded in the line scan mode across the entire device repetitively as the device was subjected to external biasing up to $14 \mathrm{~h}$ continuously. Figure 7 also depicts several line scan spectra of the Au4f region under the $+3 \mathrm{~V}$ bias, where the $\mathrm{x}$-axes correspond to the binding energy in electron volts and the y-axes correspond to the distance in millimeters from the polarized electrode toward the grounded one, recorded at the beginning, after $3.5,6.0$, and $14 \mathrm{~h}$. The color bar indicates the variations in the normalized intensity, with respect to that at the beginning $(t=0)$ of the corresponding Au4f doublet.

The Au4f spin-orbit doublet appears at 84.0 and $87.7 \mathrm{eV}$ when both electrodes are grounded, but shifts to 87.0 and $90.7 \mathrm{eV}$ 
$t=0$

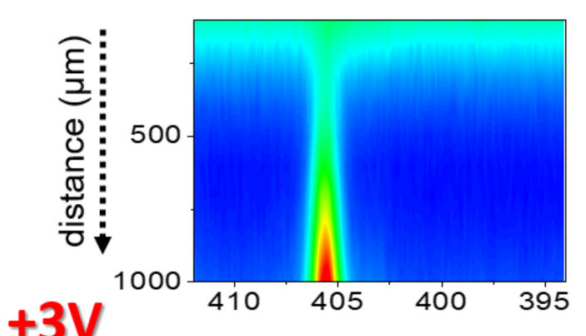

$+3 \mathrm{~V}$

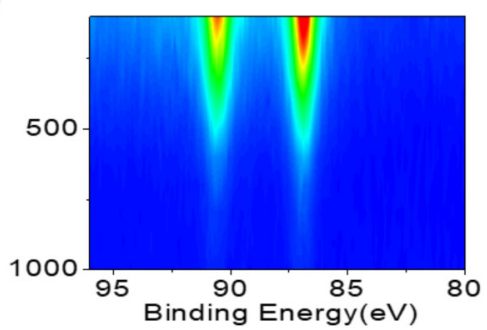

$t=4$ hours
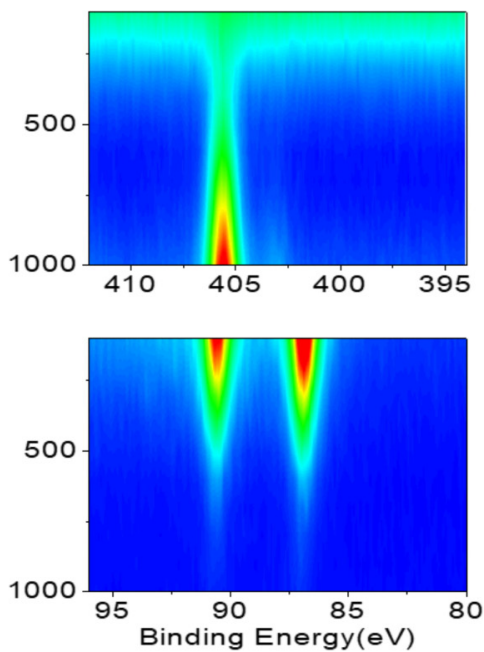

$\mathrm{t}=7$ hours

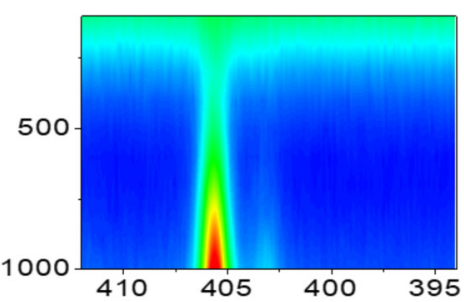

N1s

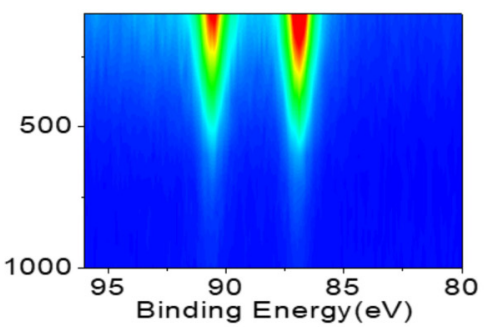

Au4f

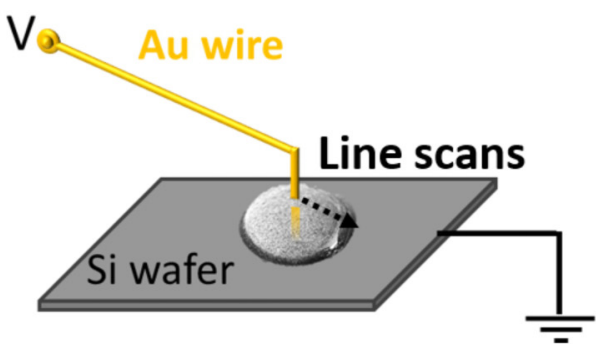

$t=12$ hours
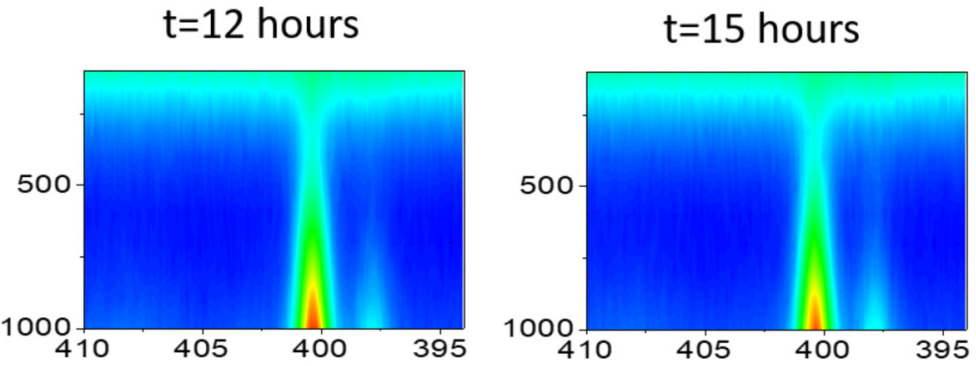

N1s

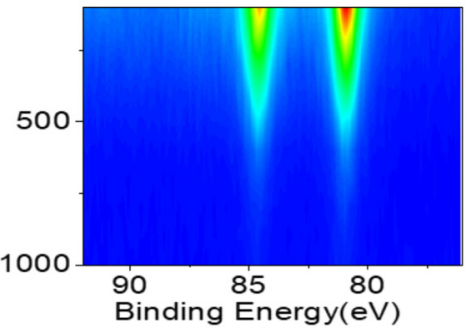

Au4f

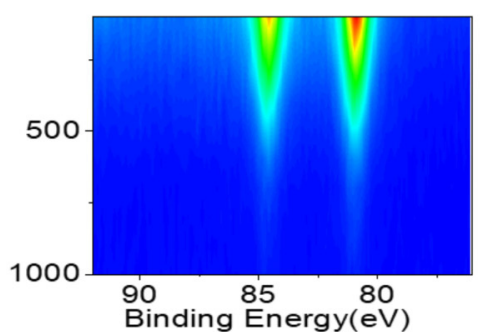

FIG. 5. N1s and Au4f regions during the course of electrochemical reduction and under $+3 \mathrm{~V}$ (top) and $-3 \mathrm{~V}$ (bottom) external bias. Reprinted with permission from Aydogan Gokturk et al., Electrochim. Acta 234, 37 (2017). Copyright 2017, Elsevier. 


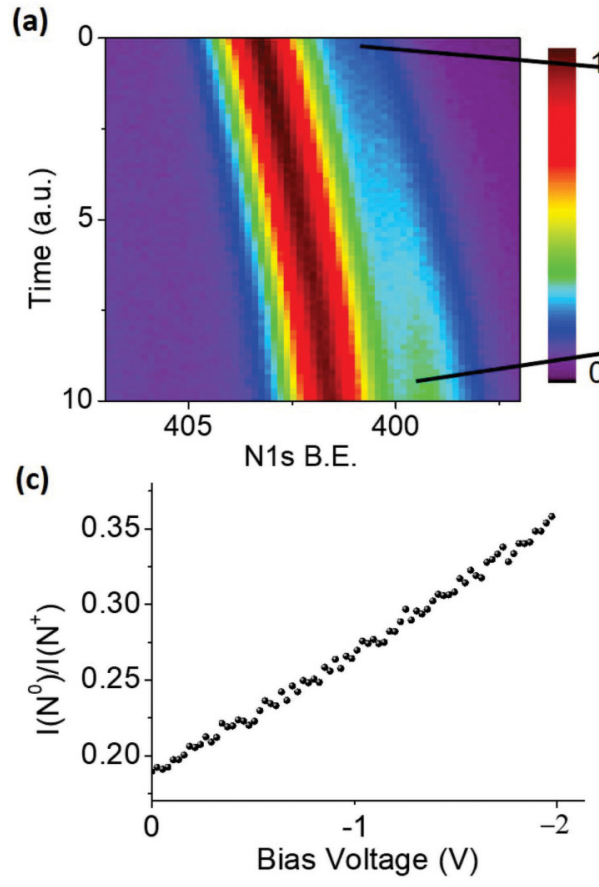

(b)

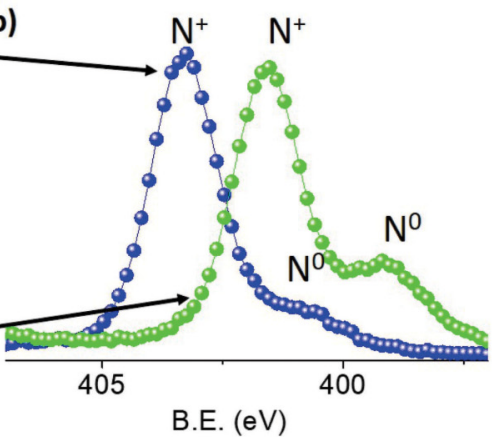

(d)

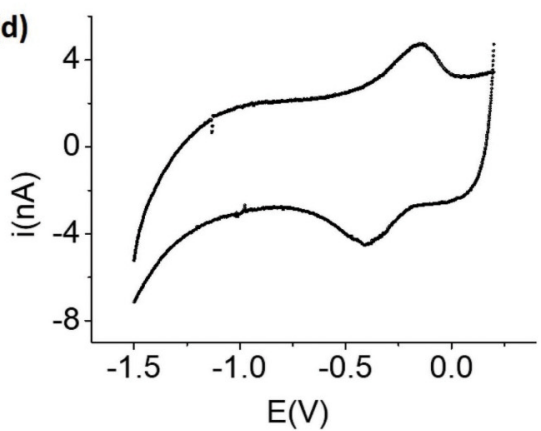

FIG. 6. (a) and (b) XP spectra of the $\mathrm{N} 1 \mathrm{~s}$ region during the first half of the electrochemical cycling. The spectrum from earlier times shows less negative binding energy shifts $(\sim-0.05 \mathrm{~V})$ compare to the one from later times $(\sim-1.9 \mathrm{~V})$. (c) Change in the ratio of the intensity of the reduced neutral nitrogen to that of the cationic ones, as a function of the applied potential. (d) Two electrode cyclic voltammogram of the system. Reprinted with permission from Aydogan Gokturk et al., Electrochim. Acta 234, 37 (2017). Copyright 2017, Elsevier.
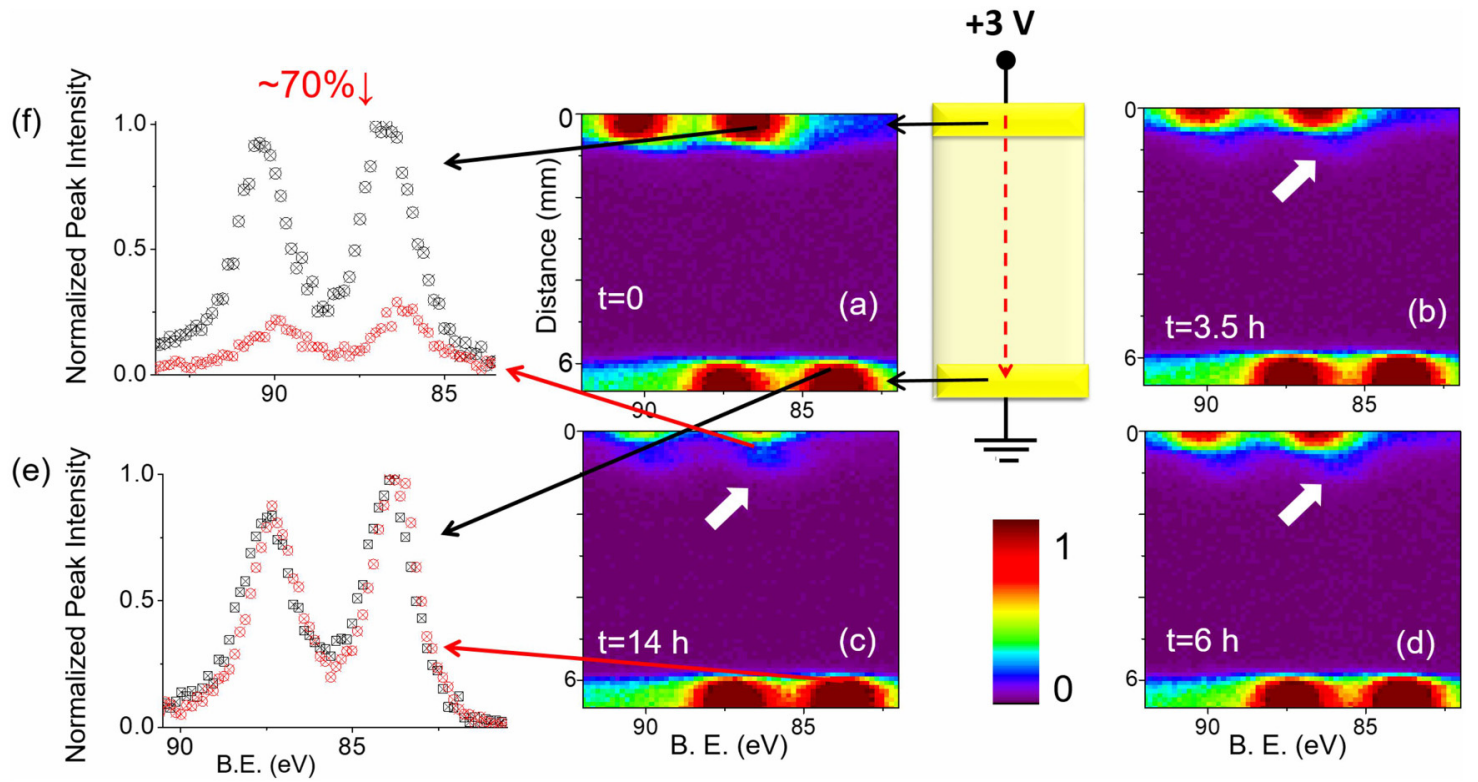

FIG. 7. Au4f XP spectra, recorded in the line scan mode from the source toward the drain electrodes; (a) at the beginning $(t=0)$, (b) after $3.5 \mathrm{~h}(\mathrm{t}=3.5 \mathrm{~h}),(\mathrm{d}) 6.0 \mathrm{~h}$ $(\mathrm{t}=6 \mathrm{~h})$, and $(\mathrm{c}) 14 \mathrm{~h}(\mathrm{t}=14 \mathrm{~h})$ during the continuous application of $+3 \mathrm{~V} \mathrm{DC}$ external potential together with the schematic representation of device and line scan direction. The color bar represents the peak intensities. Normalized Au4f spectra, with respect to those at the beginning $(t=0)$, from the two points on the line at two different positions; (f) at $500 \mu \mathrm{m}$ and (e) at $6000 \mu \mathrm{m}$ away from the polarized electrode. Adapted with permission from Camci et al., ACS Omega 2, 478 (2017). Copyright 2017, American Chemical Society. 
at the polarized one, under $+3 \mathrm{~V}$ bias. The intensity of the Au4f doublet at the source electrode decreases throughout the measurement, as shown in Fig. 7(f), whereas it is virtually the same at the drain side [Fig. 7(e)]. At the same time, a new Au species starts ascending from the source side with low intensity. On the contrary, neither a different ionic species nor a measurable electrode corrosion is detected by XPS at the grounded electrode. The corrosion is emphasized by comparing the intensity of the Au4f peaks recorded at a particular position $\mathrm{d}=0.5 \mathrm{~mm}$ from the polarized electrode, at the beginning of the measurement and after $14 \mathrm{~h}$. The normalized spectra shown in Fig. 7(f) confirms the corrosion by the significant decrease $(\sim 70 \%)$ in the intensity of Au4f photoelectron peaks after $14 \mathrm{~h}$. However, the spectra, recorded at the position $d=6.0 \mathrm{~mm}$ near the drain side of the device Fig. $7(\mathrm{~h})$, display almost no change.

To elucidate the chemical nature of the new $\mathrm{Au}$ species formed, we zoomed in on the interface and recorded data using a smaller $\mathrm{x}$-ray spot size of $50 \mu \mathrm{m}$, and recorded line scan Au4f spectra with $50 \mu \mathrm{m}$ step sizes for about a distance of $\sim 1 \mathrm{~mm}$ while imposing +3, 0, and $-3 \mathrm{~V}$ DC bias, as depicted in Fig. 8(a).

Although the secondary Au species appear as a tail of the electrode, and their spectral positions cannot be separated from those of the metallic Autf ones, when the device is grounded, they get completely separated under both positive and negative biases. In Fig. 8(c), two Au4f spectra that represent two different points on the line scan are shown. Spectra coming from the drain electrode correspond to the metallic Au4f of the Au electrode at the 2nd position on the line, and the green spectra correspond to the new Au species at the 11th position on the line. Note also that the intensities of the Au4f peaks of the new Au species, as shown both in Figs. 8(b) and 8(c), are significantly smaller. The spectral separation is related to the charging property of the surrounding medium of the new Au species, which, in actuality, is the IL medium itself. Therefore, it is best to correlate the peak positions to the F1s peak of the IL, which are also shown in Fig. 8(a).
As was pointed out in our previous work, as a result of the symmetric voltage drop at the two Au-IL interfaces, the entire IL medium retained only half of the applied potential, which was also uniform within the IL medium. ${ }^{33}$ Hence, throughout the entire IL medium surface, the F1s experienced approximately only +1.5 and $-1.5 \mathrm{eV}$ shifts (one-half of the full bias) from the grounded position when +3.0 and $-3.0 \mathrm{~V}$ bias were applied. As we will also show in Sec. IV A, the intimate correlation of the measured potential drop of the Au NPs created by electrocorrosion with that of the IL medium is also observable in the double-layer response of the coplanar capacitor geometry of the device. ${ }^{34}$ In Fig. 9, we give an emphasized representation of this finding.

As a conclusion to this section, we claim we had demonstrated that Au NPs can be synthesized by electrochemical oxidation, possibly first by the generation of $\mathrm{Au}^{+1}$ ions, which are reduced to $\mathrm{Au}^{0}$ and coalesce within the DEME-TFSI medium. The charging/discharging properties of the Au metal is different from those of the $\mathrm{Au}$ NPs dispersed in the IL medium, leading to completely different responses with the electrical excitations, hence offering a perfect spectral separation.

\section{Electrochemically induced intercalation of an ionic liquid into graphene multilayers}

This section summarizes our work on utilizing XPS to monitor in situ the changes in the anion/cation intensity ratio of the same DEME-TFSI ionic liquid, under applied electric fields, which induces intercalation. ${ }^{62}$ We also measured the electrical potential developments on different surface structures of a multilayered graphene assuming the role of the top electrode. These potential developments were derived from the apparent shifts in the binding energies of the corresponding atomic core levels in a chemically resolved fashion. $^{21,63}$

A PEM separator with $43 \%$ porosity was used to allow the IL to pass but prevented shorting of the top multilayer graphene (a)

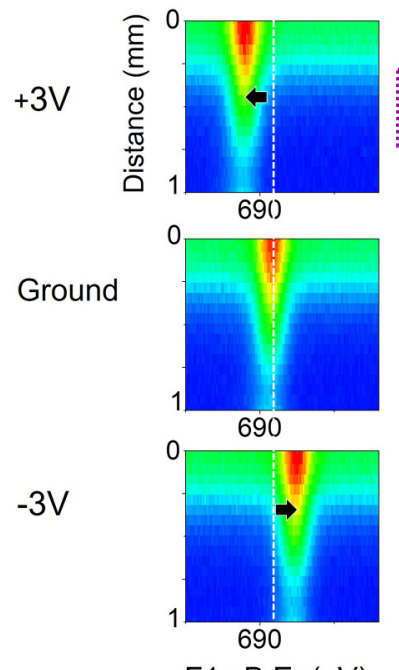

F1s B.E. (eV) (b)
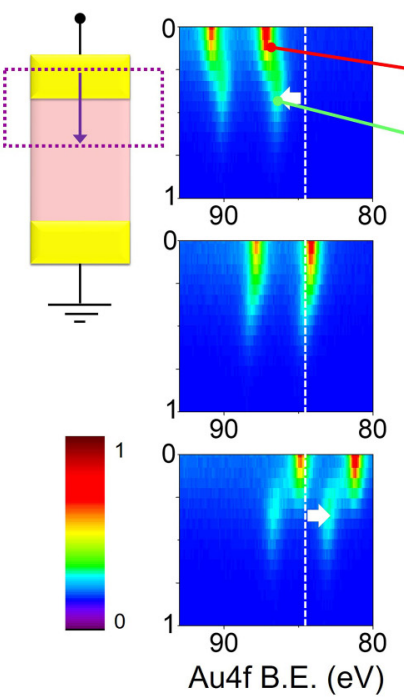

(c)

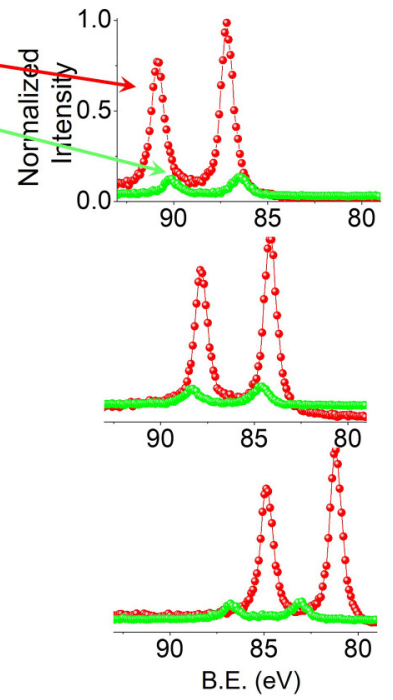

FIG. 8. XP spectra of (a) F1s and (b) Au4f region in the line scan mode along $1 \mathrm{~mm}$ from the source electrode, recorded under application of $+3,0$ (grounded), and $-3 \mathrm{~V}$ DC potential. (c) Au4f XP spectra recorded in a higher resolution (normal scan mode) on Au electrode and at interfacial region. Adapted with permission from Camci et al., ACS Omega 2, 478 (2017). Copyright 2017, American Chemical Society. 


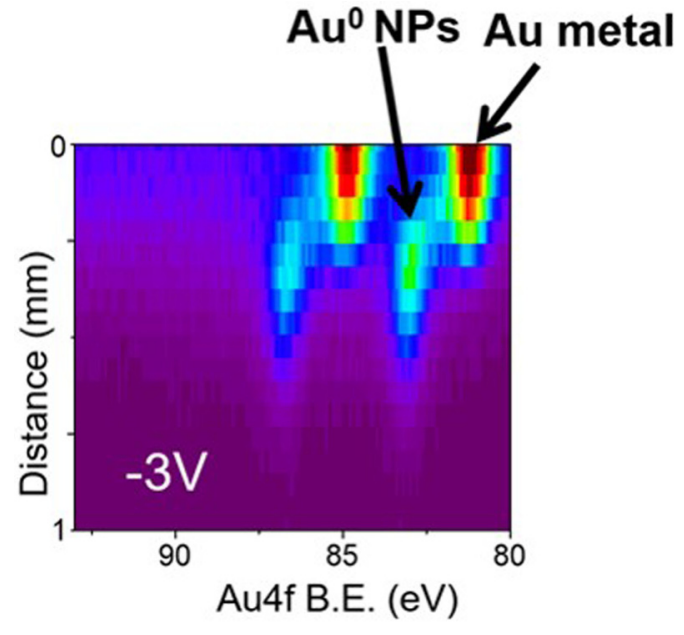

FIG. 9. Line scan spectra of the Au4f representing the two different Au species when $-3.0 \mathrm{~V}$ bias was applied. Adapted with permission from Camci et al., ACS Omega 2, 478 (2017). Copyright 2017, American Chemical Society.

(MLG) and the bottom gold electrodes. Multilayer graphene samples were grown on nickel foils (Alfa Aesar) using chemical vapor deposition. This process resulted in fabricating multilayer graphene samples having 100-250 layers. ${ }^{64}$ The MLG electrode was transferred onto the PEM and is directly placed on the gold electrode. The ionic liquid was initially introduced in between the PEM and the bottom electrode, as schematically shown in Fig. 10. A survey spectrum of the IL, together with the detailed F1s, N1s, and $\mathrm{C} 1 \mathrm{~s}$ regions, are also shown in the same figure. The N1s region consists of two equally intense and well-resolved peaks representing the cationic and the anionic fragments of DEME-TFSI. The F1s region has only one peak and $\mathrm{C} 1 \mathrm{~s}$ has several.

Since the XP spectra were recorded from the top MLG electrode, none of the peaks belonging to the IL was observable at the beginning. However, when a suitable voltage bias was applied, the intercalation process became operative and asymmetric ion movement of the IL through the MLG electrode was detected as depicted in Fig. 11.

As can be gathered from Fig. 11, upon application of a positive bias from the bottom gold electrode, the intensity of the cationic N1s peak dominates, and the situation is reversed for the negative bias, revealing for the first time a chemical and visual demonstration of the intercalation process, using XPS. This recorded asymmetry in the cationic/anionic ratio differs from unity by as much as $10 \%$ going up or down depending on the polarity imposed.

Additionally, the bias-dependent binding energy shifts followed through atomic features of the IL (F1s, N1s, and C1s) and the graphene electrode $(\mathrm{Cls})$ indicate that a distinct solid-liquid interface develops throughout the entire intercalation process with an additional and pertinent evidence for finite potential drops across the two electrical double layers. As summarized in Fig. 12, this finding is bolstered by the fact that the measured binding energy difference between the F1s of the liquid and C1s peak of the semisolid MLG electrode is only about half of the applied bias,
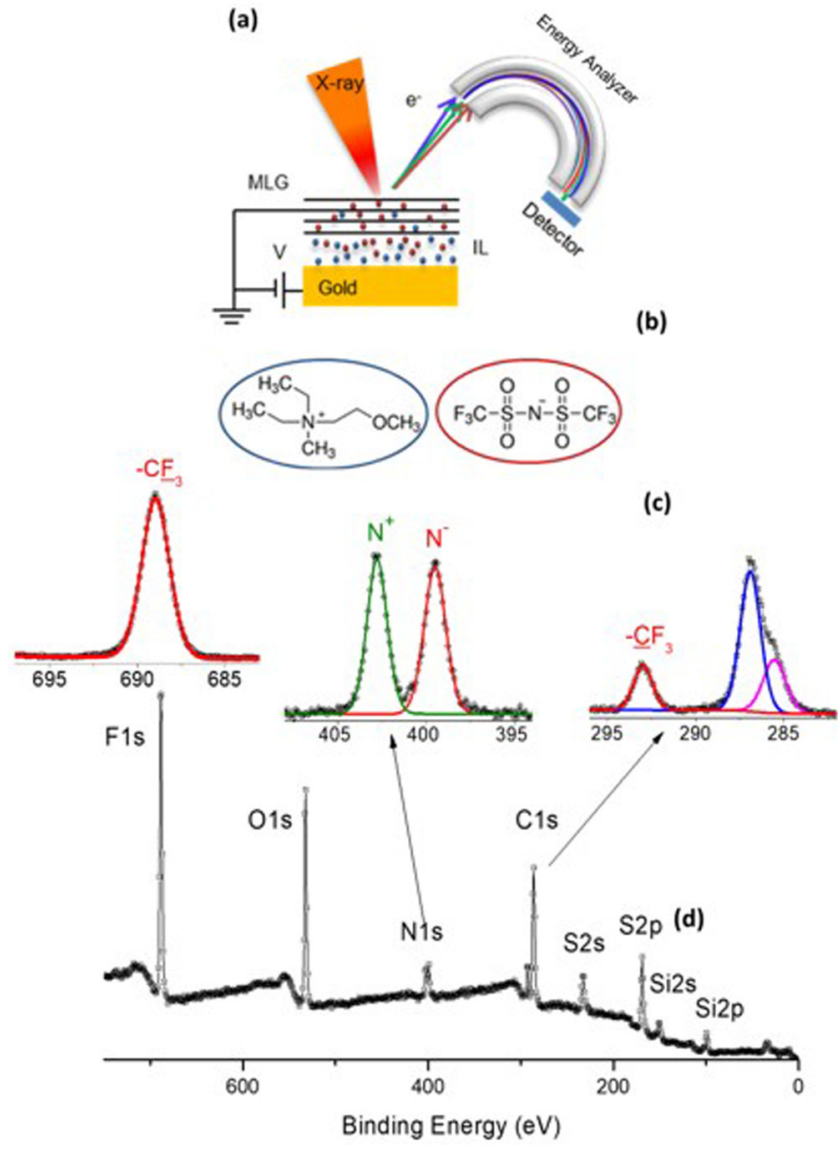

FIG. 10. (a) Experimental setup used for the operando XPS in the back-gate geometry. (b) Chemical structure of the ionic liquid used. (c) Selected core levels. (d) Survey XP spectra. Adapted with permission from Camci et al., J. Phys. Chem. C 122, 11883 (2018). Copyright 2018, American Chemical Society.

where the rest of the applied voltage is screened by the two electrical double layers at the solid-liquid interfaces between (i) the MLG-IL and (ii) IL-bottom metal electrode.

We can summarize the important findings of this section as follows. We utilized two important assets of XPS for (i) quantification of the nonstoichiometric ion movement/intercalation through the pores of a multilayer graphene top electrode in response to voltage bias, and (ii) mapping of the electrical potential developments on the graphene electrode intercalated with the IL, in a totally noninvasive and chemically specific fashion

\section{AC ACTUATION USING SQUARE-WAVES AND ITS APPLICATIONS}

In order to probe/underpin time-dependent properties of the samples under investigation, it is necessary to impose a timedependent (AC) actuation. For electrical and electrochemical measurements, the natural choice is a sine-wave with discrete or continuously varying frequencies. As will be shown later, sine-wave 
C1s

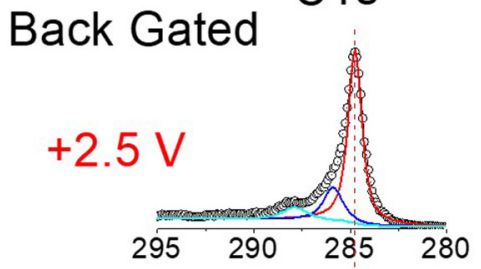

N1s

$\mathrm{N}+$

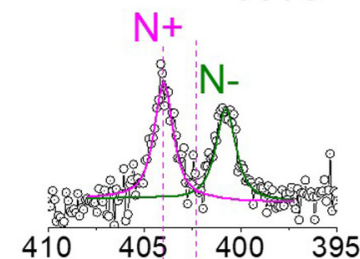

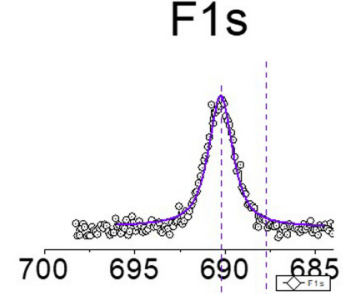
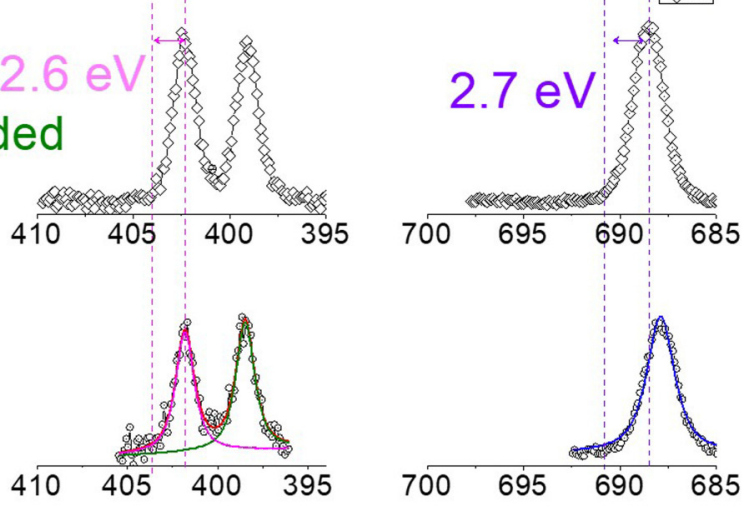

Binding Energy (eV)

FIG. 11. C1s, F1s, and N1s regions of the intercalated thick graphene layer with the $\mathrm{IL}$ at $+2.5,0 \mathrm{~V}$ (grounded) and $-2.5 \mathrm{~V}$ back-gate voltages. Adapted with permission from Camci et al., J. Phys. Chem. C 122, 11883 (2018). Copyright 2018, American Chemical Society.

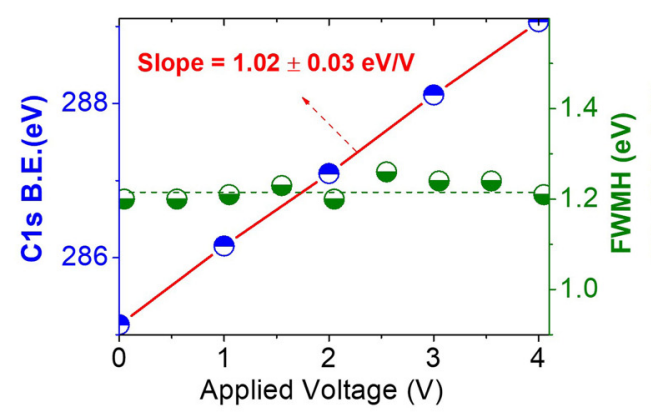

(a) $\mathrm{C} 1 \mathrm{~s}$

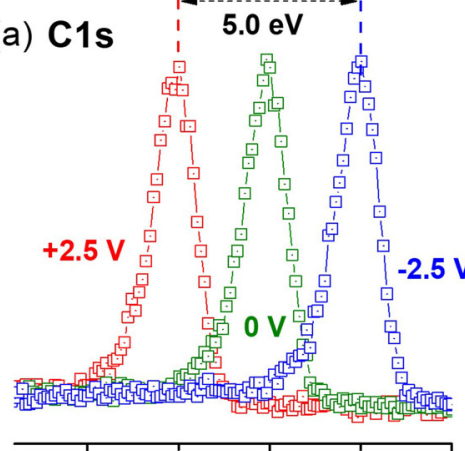

290

285

\section{0}

B.E. $(\mathrm{eV})$

FIG. 12. (a) C1s region of the multilayered graphene top electrode under $+2.5,0$ (grounded), and $-2.5 \mathrm{~V}$ bias, where the shifts in the binding energy positions comply exactly with the applied bias. (b) F1s region recorded from the surface of the device under bias, changing from 0 to $+4 \mathrm{~V}$. Insets show linear fits (line) of the position and the full-width half-maximum of the C1s and F1s (IL on MLG electrode) peaks against the applied bias. For the linear fit of $\mathrm{F} 1 \mathrm{~s}$, points at 0 and $4 \mathrm{~V}$. Adapted with permission from Camci et al., J. Phys. Chem. C 122, 11883 (2018). Copyright 2018, American Chemical Society. 
actuation distorts the form of the XP signal. Therefore, we have adopted the use of square-wave (SQW) actuations instead.

\section{A. Probing double-layer properties of a coplanar capacitors involving an ionic liquid using ultralow frequency actuation}

This section deals with application of an ultraslow $(10 \mathrm{mHz}) 5 \mathrm{~V}$ AC SQW excitation of the same device as described in Secs. III A and III B. This procedure enabled us to study the charge screening dynamics with both temporal and lateral resolution, as shown in Fig. 13. The F1s spectrum was recorded iteratively with the snapshot mode and with $1 \mathrm{~s}$ intervals at nine different positions along the $\sim 8 \mathrm{~mm}$ long device.

Temporal resolution was attained through recording the response of the surface potential extracted from the equally slow variations in the binding energy position of the F1s peak representing the anion of the IL, and the lateral resolution was achieved by recording the same spectra throughout the different lateral position with $800 \mu \mathrm{m}$ intervals along the entire electrochemical device. Each time-resolved frame consists of 300 spectra, during which the F1s peak experiences six polarity $( \pm 5 \mathrm{~V})$ changes. Upon a single polarity change, the position of the F1s peak moves up or down in binding energy scale, depending on its distance from the polarized electrode, and slowly returns to either +2.5 or $-2.5 \mathrm{~V}$ displacement form its grounded position of $688.5 \mathrm{eV}$, as was also mentioned in Sec. III and represented in Figs. 3(a) and 3(b).

This temporal behavior is attributed to the screening of the imposed electrical potential by the ions and the formation of the oppositely charged EDL in the vicinity of the two metal electrodes. The lateral behavior is even more informative, since the F1s signal shows a complete reversal with respect to its position, faithfully
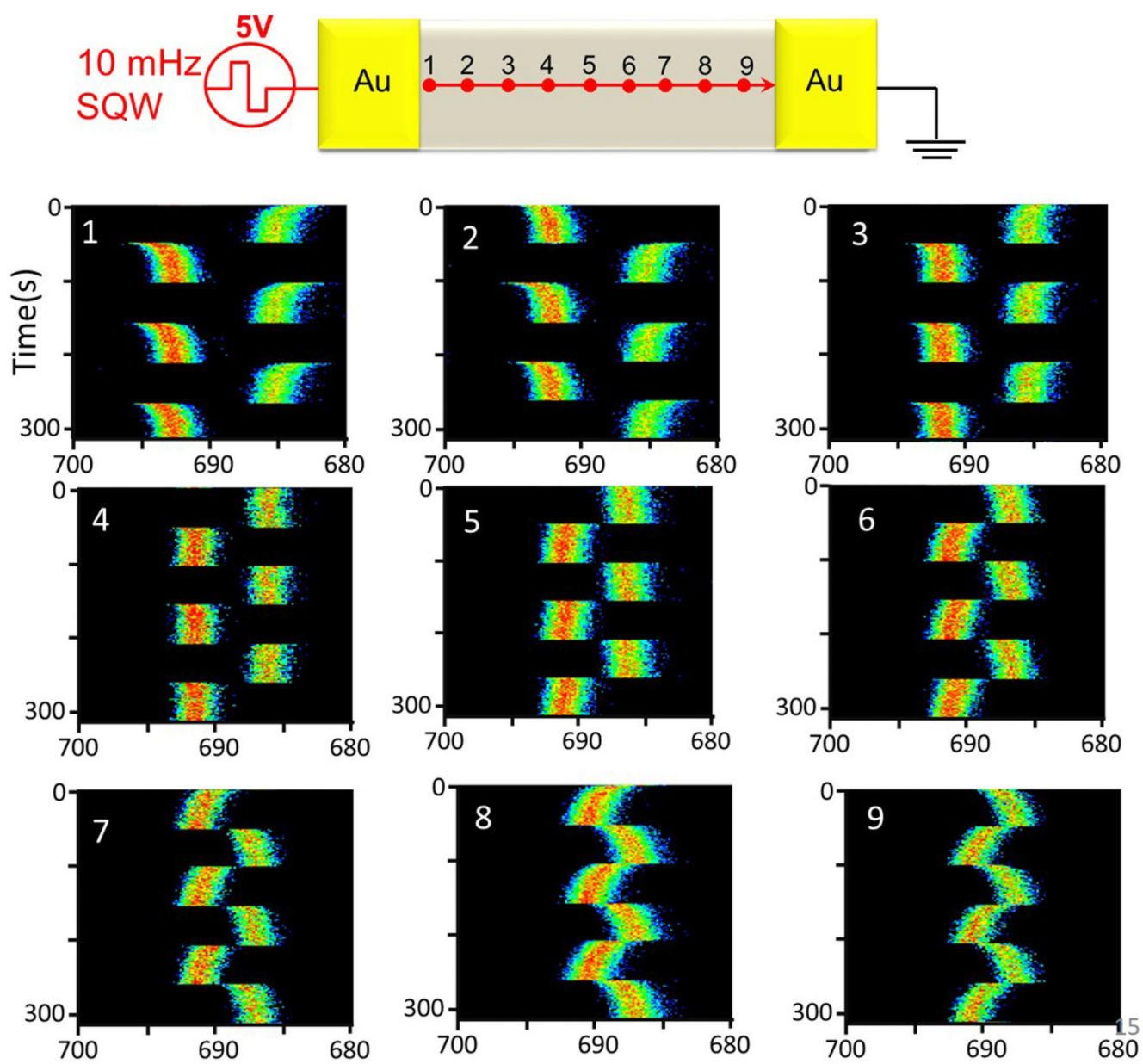

F1s Binding Energy (eV)

FIG. 13. Time-resolved F1s XP spectra recorded at nine different lateral positions across the metal electrodes with $800 \mu \mathrm{m}$ step size between each point. Adapted with permission from Camci et al., Phys. Chem. Chem. Phys. 18, 28434 (2016), Copyright 2016, PCCP Owner Societies. 
following the proposed EDL model with two oppositely charged electrode/IL interfacial regions and a hypothetical neutral midpoint. Upon imposition of the voltage stimuli between the Au electrodes, the EDL forms because the mobile ions of the IL screen the local voltage on the electrode, and this charge screening starts its action in the opposite directions at the other Au electrode. Since mobile ions screen in opposite rearrangement directions for two $\mathrm{Au}$ electrodes at each end, there exists a point of no motion near middle of the IL-device, and this hypothetical point is consistent with the experimental findings represented in Fig. 13, as the response of point- 1 is totally reversed in point- 9 , whereas at the point- 4 the device is nonresponsive.

This surprising finding surfaces an important point that although the EDL formation is extremely short-range, in orders of tens of nano-meters, its electrical response is transmitted throughout unprecedented distances in the millimeter range.

This newly established temporal and lateral behaviors of the EDL can also be detected by other species created and/or embedded into the IL medium, such as Au NPs, mentioned in Sec. III B, as summarized in Fig. 14.

In this figure, there are three physically and/or chemically distinct neutral $\mathrm{Au}^{0}$ species; $\mathrm{Au}$ metal at (i) the polarized- and (ii) the grounded electrodes, and (iii) Au NPs surrounded by IL moieties. When the electrodes are both grounded, all three species are detected at $84.0 \mathrm{eV}$ binding energy. However, upon imposing the $10 \mathrm{mHz}$ and $5 \mathrm{~V} \mathrm{AC}$ (square-wave) excitation, their binding energies become differentiated in time. Depending on the sign of the polarization, the position of the Au4f doublet shifts with the +5 and the $-5 \mathrm{~V}$ promptly, whereas no such a difference can be detected on the grounded electrode-side. The most intriguing observation is that the Au4f doublet tracts faithfully both the temporal and lateral variations as the F1s peak of the IL, throughout the same extremely long distances.

This latter finding has important implications toward improvement in the sensitivity of spectral analyses of complex liquids and their interfaces, where signal identification might not be so straightforward using XPS, since Au4f peaks have notoriously large cross sections and are, therefore, easy to detect.

\section{B. Impedance like actuations and a new perspective to investigate electrowetting phenomena}

Electrowetting on dielectrics (EWOD) is a heavily investigated phenomenon, utilized within a variety of scientifically and technologically important areas, such as microfluidics, liquid lenses and displays, biochemical separation and analytical sciences, etc. ${ }^{65-73}$ Electrowetting is simply the control of wettability of liquids by application of electrical stimuli, in DC and/or AC forms. ${ }^{74}$ This process had been investigated using a number of electrochemical, spectroscopic, and microscopic tools, ${ }^{75}$ but the use of XPS was sparse. ${ }^{76-78}$ Within the last three years, we have reintroduced XPS as a powerful analytical arsenal for probing electrical potential developments of liquids and their interfaces with dielectric substrates to extract novel information about them, under in situ and in operando conditions. ${ }^{37-40}$

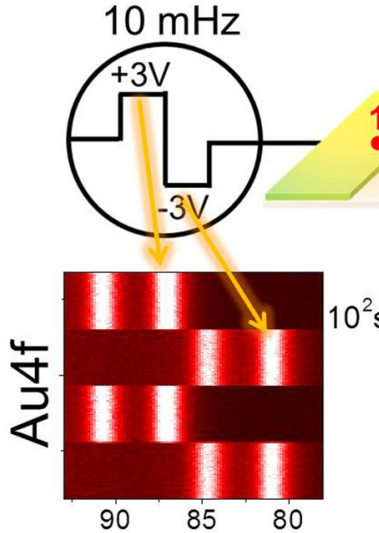

(1)

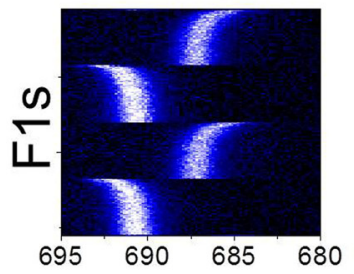

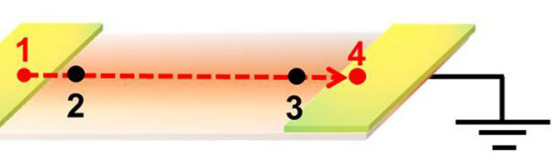

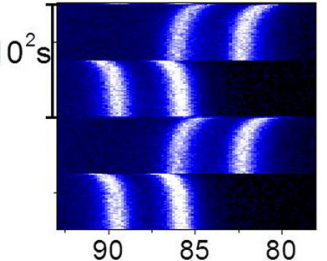

(2)

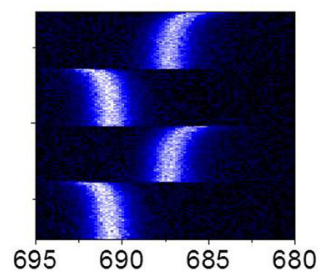

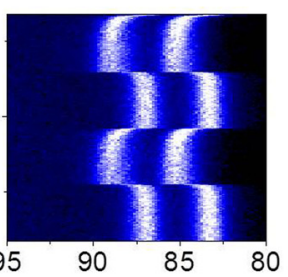

(3)

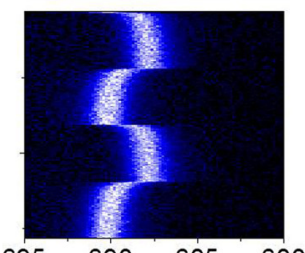

$695690 \quad 685 \quad 680695$
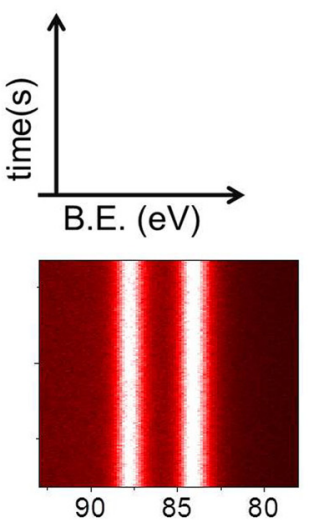

(4)

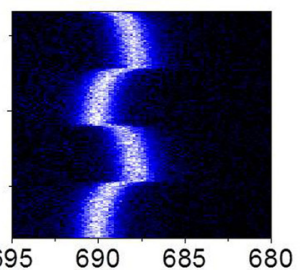

FIG. 14. Time-resolved XP spectra of Au4f and F1s regions under $10 \mathrm{mHz}$ SQW excitation and recorded at four different lateral positions. Adapted with permission from Camci et al., ACS Omega 2, 478 (2017). Copyright 2017, American Chemical Society. 


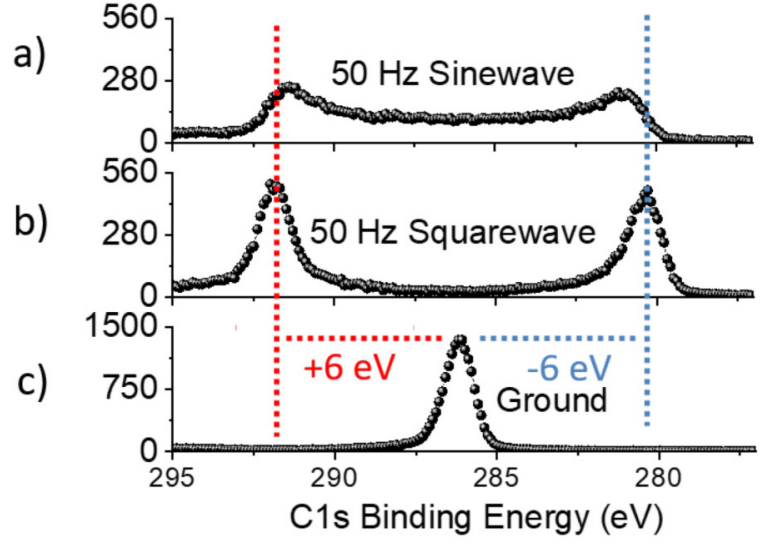

d)

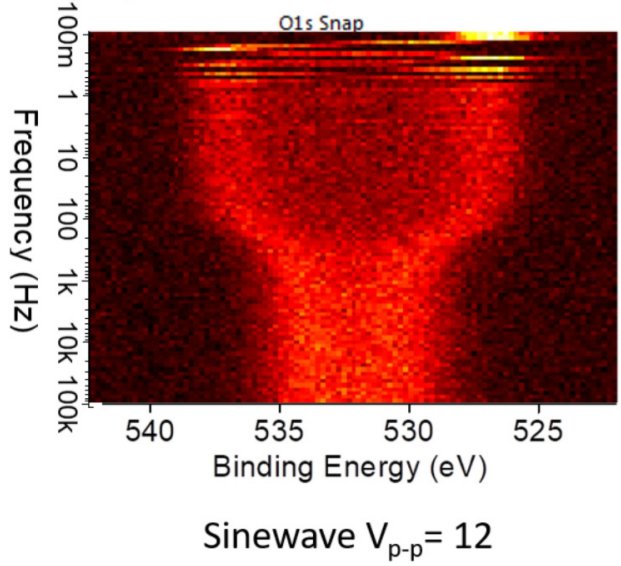

e)

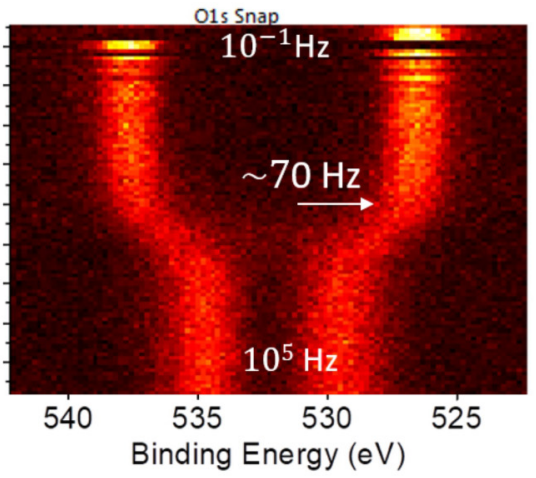

Squarewave $V_{p-p}=12$
FIG. 15. $C 1 \mathrm{~S} X P$ spectra of PEG when the wire electrode is subjected to (a) sine wave, (b) square wave excitation of $6 \mathrm{~V}$ amplitude with $50 \mathrm{~Hz}$ frequency and (c) grounded while the substrate is grounded. Time-dependent iterative spectra of 01 s region as a $3 \mathrm{D}$ plot where each row represents a single spectrum under $6 \mathrm{~V}$ (d) sine-wave and (e) square-wave starting from the frequency of $100 \mathrm{mHz}$ till $100 \mathrm{kHz}$, and changes in a logarithmic fashion. Adapted with permission from Aydogan Gokturk et al., Langmuir 35, 3319 (2019). Copyright 2019, American Chemical Society.
In order to probe impedance type properties of the samples under investigation, it is necessary to impose a varying frequency (AC) actuation for which the natural choice is a sine-wave. However, as we said earlier, the sine-wave distorts the form of the XP signal, see Figs. 15(a) and 15(d). Therefore, we have adopted the use of square-wave actuations instead, which causes only twining of all the peaks at the minimum and the maximum of the applied voltages, as is also shown in the same Fig. 15(b). However, we have also checked and ensured that the crucial information derived from analyses of such data, such as the critical frequency (to be described next), is the same in both cases, see Figs. 15(d) and 15(e).

In the same figure, $\mathrm{O} 1 \mathrm{~s} \mathrm{XP}$ spectra recorded while imposing a logarithmic sweep of certain range $\left(10^{-1}-10^{5} \mathrm{~Hz}\right.$ in this case) are also shown, which enables us to follow the surface potentials by recording the O1s signal at various points on the droplet surface. When actuated from the wire electrode, the PEG, a dielectric liquid, adopts the potential of the inserted wire electrode at very low frequencies up to $\sim 70 \mathrm{~Hz}$, and two O1s peaks reflecting the positive and the negative components of the SQW potential bias are measurable at +6 and $-6 \mathrm{eV}$ positions, i.e., $12 \mathrm{eV}(\triangle \mathrm{ABE})$. After $70 \mathrm{~Hz}$, the difference in the binding energy positions of these two peaks decreases continuously and reaches a steady state value at $\sim 800 \mathrm{~Hz}$, which is maintained at higher frequencies. At very low frequencies, the droplet behaves like a conductor; hence, the entire voltage drop occurs in the dielectric layer. At high frequencies, it behaves like a perfect dielectric. The critical frequency between these two behaviors is determined by the following equation: ${ }^{74,75,79}$

$$
f_{c}=\frac{\sigma_{l}}{2 \pi\left(\varepsilon_{l}+\varepsilon_{d} \frac{R}{d}\right)},
$$

where $\varepsilon_{l}$ and $\varepsilon_{d}$ are the electrical permittivity of the liquid droplet and the dielectric layer, respectively; $\sigma_{l}$ is the conductivity of the liquid; $R$ is the radius of the droplet; and $d$ is the thickness of the dielectric layer. Beyond the critical frequency, a fraction of the potential that is applied to the wire drops within the droplet and results in the decrease of the effective contact angle change.

The measured steady state potential at higher frequencies depends strongly on the position and adapts to a fraction of the applied potential, as a function of the distance of the analyzed point from the wire electrode, as depicted in Fig. 16, which show iterative O1s signals recorded at the three different and indicated positions on the drop.

As revealed by the figure, while the lower critical frequency stays constant, the amplitude of the potential measured varies with 


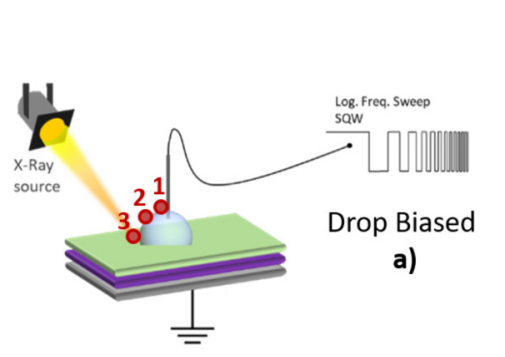

1
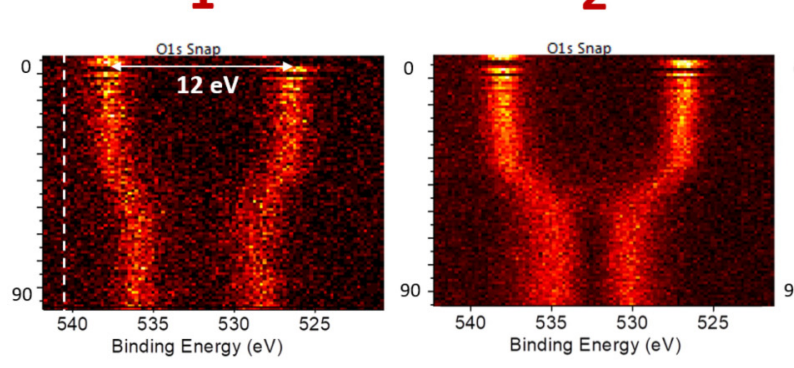

2
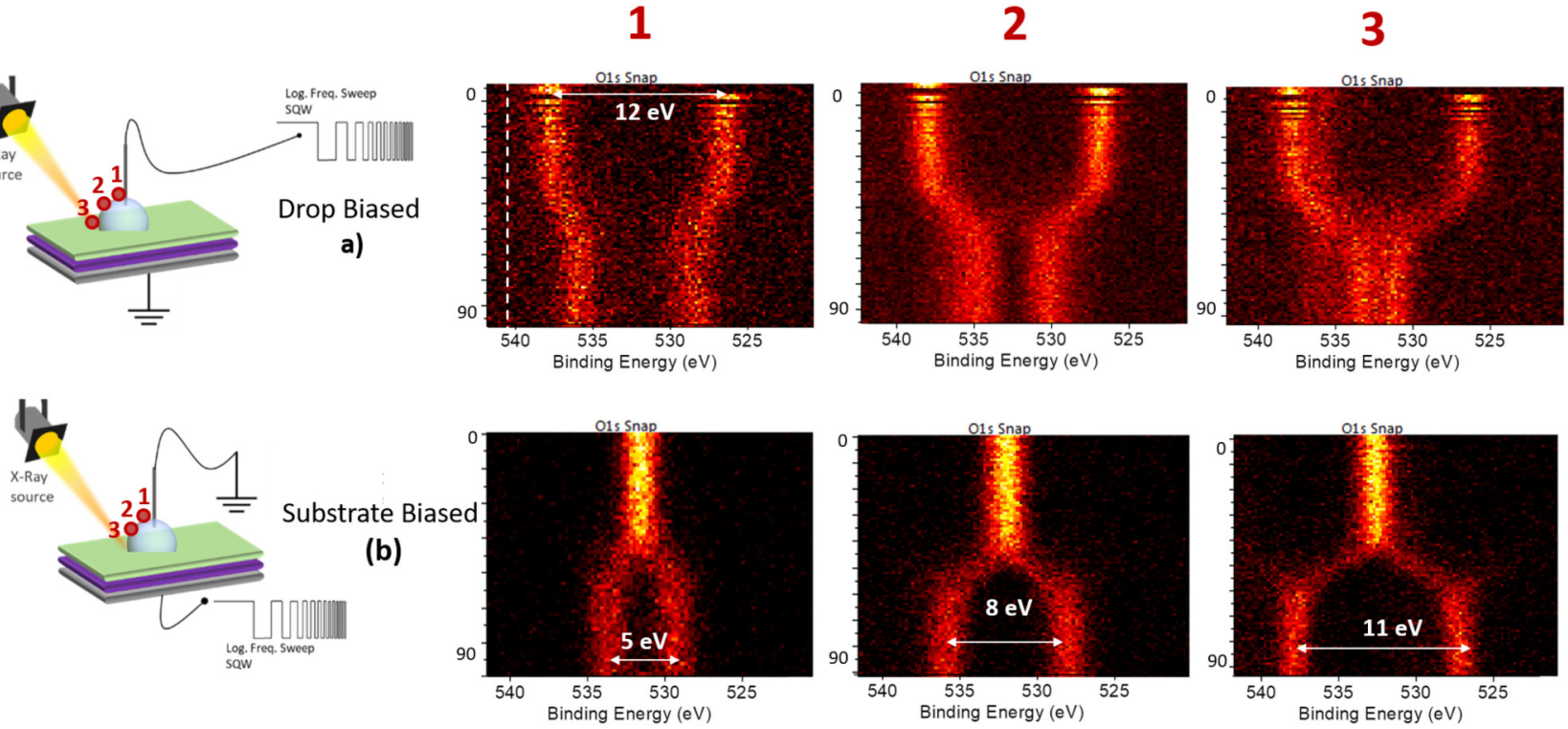

FIG. 16. Schematic representation of the $A C$ actuation by imposing the bias from (a) the top or (b) the bottom electrodes, together with the recorded $01 \mathrm{~s}$ spectra at three different lateral positions on the drop, while sweeping the frequency. Adapted with permission from Aydogan Gokturk et al., Langmuir 35, 3319 (2019). Copyright 2019, American Chemical Society.

the distance from the electrode at higher frequencies. At a point close to the liquid/dielectric interface, negative and positive components of the O1s peak merges to each other (near zero IR drop), as opposed to the lower frequency regime, where no IR drop is measurable anywhere on the liquid, i.e., always a full $12 \mathrm{eV}$ separation between the two components is measured.

\section{Extracting equipotential lines on a liquid drop}

In order to extend these measurements to cover the entire surface of the liquid drop, we recorded the O1s signal in the areal mapping mode and at only two frequencies representative of the lower and the higher frequency regimes (lower and higher than the critical frequency), since collecting areal data with varying frequency at every point is not feasible. Afterward, we fitted the XP spectrum at each point to two Ols peaks, corresponding to the negative and the positive components and took the binding energy difference between them. The extracted binding energy differences of two components are displayed in Fig. 17 as areal maps for the lower $(50 \mathrm{~Hz})$ and higher $(1 \mathrm{kHz})$ frequencies separately.

One can clearly see that at $50 \mathrm{~Hz}$, the liquid completely screens the applied electrical field and the entire potential drop takes place at the liquid/dielectric interface. As a result, only one $\mathrm{O} 1 \mathrm{~s}$ peak is observed. But, at $1 \mathrm{kHz}$, the liquid can no longer screen the field, and the drop behaves like a resistive medium, resulting in splitting of the O1s peaks. The magnitude of the splitting is near zero at the tip of the top electrode and increases to up to $12 \mathrm{eV}$ at the liquid/dielectric interface. This behavior is evidenced by the equipotential surface contours extracted.
This section is a truly novel demonstration of the use of labbased XPS instruments for extracting frequency dependent AC potential distributions on liquid surfaces and their interfaces in a most direct and noninvasive fashion.

\section{Equivalent circuit and simulation of XP spectra}

For the discussions of this section, a slightly different EWOD geometry is used, where the liquid is the same PEG drop as the one given in Sec. IV B 1, but on a different dielectric substrate, consisting of $\sim 100 \mathrm{~nm}$ Teflon 1600 coated on $\mathrm{SiO}_{2}(\sim 300 \mathrm{~nm}) / \mathrm{Si}$. The capacitance of the dielectric under the drop was estimated as $60 \mathrm{pF}^{40}$ The results of recorded AC spectra together with the DC one are summarized in Fig. 18.

In order to compare the recorded XP spectra under AC actuation, output voltage waveforms from an electrical circuit are obtained using LT-SPICE software. ${ }^{80}$ The resulting waveforms are used to generate the recorded XP spectra by using an in-house developed PYTHON program, through convolution of a line shape function with the grounded C1s spectrum. Following the previously published work, a circuit model is constructed to describe the EWOD system under investigation electrically, as shown in Fig. 19(a). ${ }^{38}$ The PEG droplet is represented as a capacitor $\left(\mathrm{C}_{\text {liquid }}\right)$ in a parallel connection with a finite number ( 5 in this case) of resistors in series $\left(\mathrm{R}_{\text {liquid }}\right)$. In principle, one has to use an infinite number of resistors for a realistic representation of a liquid, but the finite number is chosen for practical simulation purpose(s). Furthermore, as was shown by numerous studies in the literature, the dielectric capacitance dominates the electrical response, and for a reliable dielectric, the leakage resistance is assumed to be unmeasurable, i.e., 
(a)

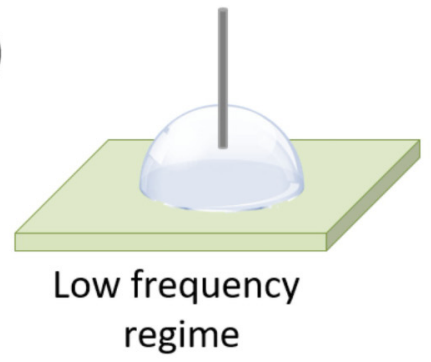

(b)

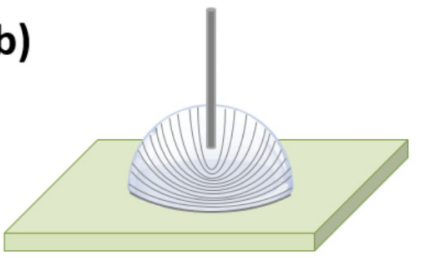

High frequency regime

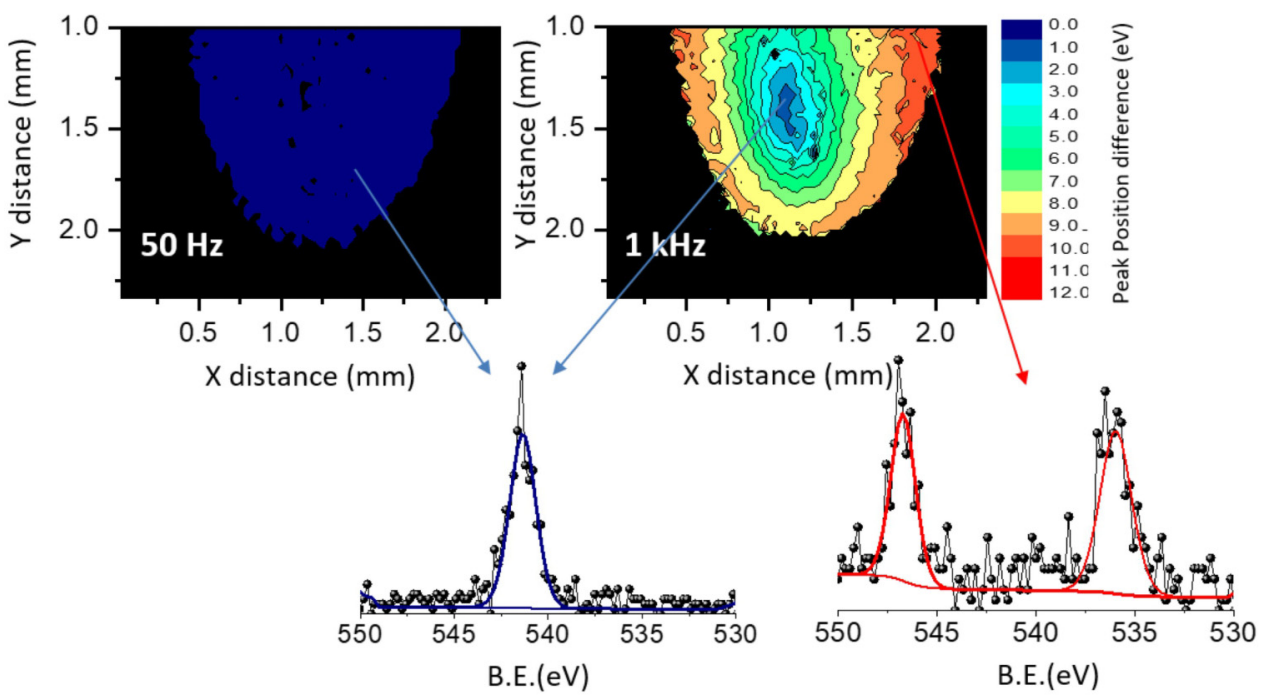

FIG. 17. Areal maps of the binding energy difference of positive and negative components of 01s under the applied $6 \mathrm{~V}$ square-wave bias from the bottom electrode with (a) $50 \mathrm{~Hz}$ and (b) $1 \mathrm{kHz}$ frequencies together with their schematic representations. Adapted with permission from Aydogan Gokturk et al., Langmuir 35, 3319 (2019). Copyright 2019, American Chemical Society.
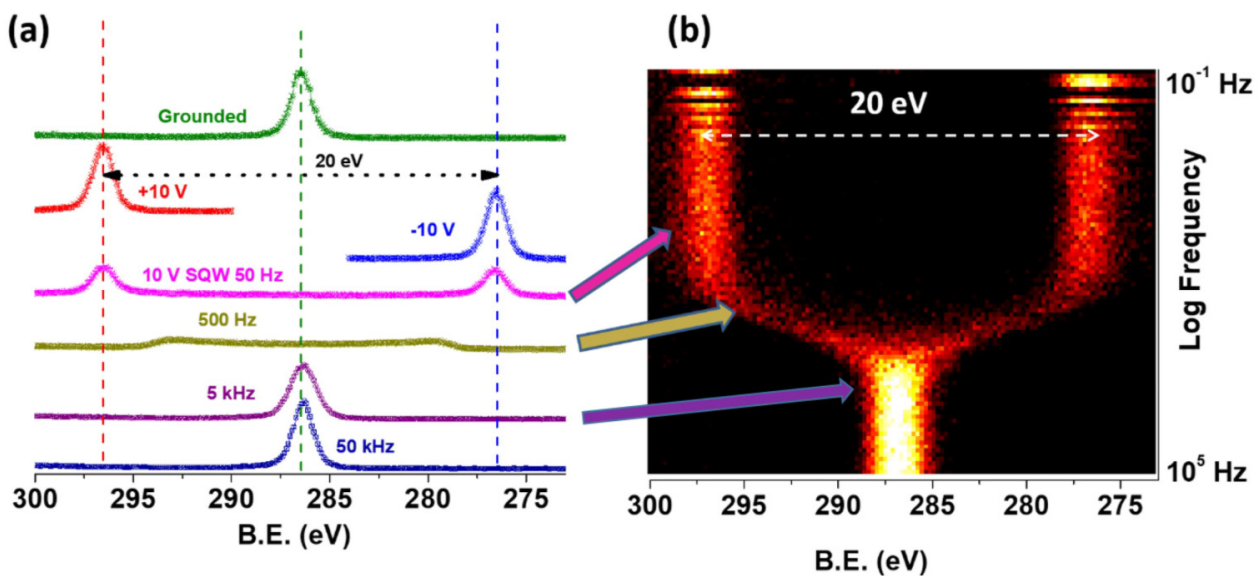

FIG. 18. (a) C1s region recorded without any bias, and under +10 and $-10 \mathrm{~V} D C$ bias, together with $10 \mathrm{~V}$ SQW AC bias at four different frequencies. (b) The same data recorded using the snapshot mode with $1 \mathrm{~s}$ time resolution, while the drop is subjected to a logarithmic frequency sweep between $10^{-1}$ (top) to $10^{5} \mathrm{~Hz}$ (bottom). Under $\mathrm{AC}$ actuations, intensity of the peak is halved since the peak is twinned. However, additional intensity changes are also encountered due to movement of the drop, which causes differences in the electron collection efficiency of the spectrometer. Adapted with permission from Aydogan Gokturk et al., Langmuir 35, 3319 (2019). Copyright 2019, American Chemical Society. 
(a)

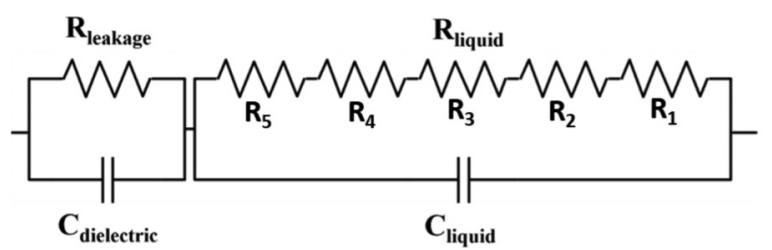

(b)

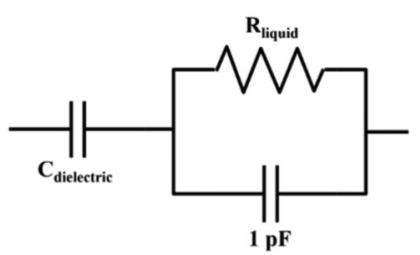

FIG. 19. (a) Equivalent circuit model for the EWOD system and (b) simplified version. Adapted with permission from Uzundal et al., Langmuir 35, 16989 (2019). Copyright 2019, American Chemical Society.

$>10 \mathrm{G} \Omega$ in our case. Therefore, the circuit is simplified further as shown in Fig. 19(b).

The critical frequency for the present system was measured as $\sim 170 \mathrm{~Hz}$, whereas it was reported to be $\sim 70 \mathrm{~Hz}$ for the system described in Sec IV B, on a similar system, indicative of a higher capacitance for that dielectric layer. ${ }^{40}$ This number is consistent because, therein, a much thinner $(\sim 50 \mathrm{~nm})$ hydrophobic coating with CYTOP was utilized, instead of the $1 \mu \mathrm{m}$ Teflon coating of this work, and taking into account that the capacitance is inversely proportional to the thickness of the dielectric layer.

Guided by the results of these simulations, together with XPS measurements on a mimicking device, fabricated by using only solid-state components, we assigned a value of $14 \mathrm{M} \Omega$ for the resistance of the liquid drop and $60 \mathrm{pF}$ for the capacitance of the dielectric layers to match the measured critical frequency of $\sim 170 \mathrm{~Hz}{ }^{79}$ The results of the simulation process, together with measured spectra, are shown in Fig. 20.

As can be seen from Fig. 20(d), this procedure and the values used faithfully reproduce the measured XP spectra recorded at 50, 500 , and $5 \mathrm{kHz}$, respectively. Furthermore, the determined resistance of $14 \mathrm{M} \Omega$ is quite reasonable, when compared to experimentally reported conductivity value $\left(\sim 10^{-7} \mathrm{~S} / \mathrm{m}\right)$ for the PEG-600 liquid. ${ }^{81}$

\section{Discovering an additional asymmetry in electrowetting}

For the EWOD process using aqueous solutions, two important obstacles are encountered at high-applied potentials; (i) contact angle saturation and (ii) dielectric failure. They have both been postulated to stem from accumulation of charges at liquid/ dielectric interface(s). An asymmetric behavior with respect to the polarity of the applied potential is another phenomenon, which has also been claimed to stem from the adsorption of specific ions at the liquid/dielectric interface. ${ }^{82,83}$ It was also claimed that the same

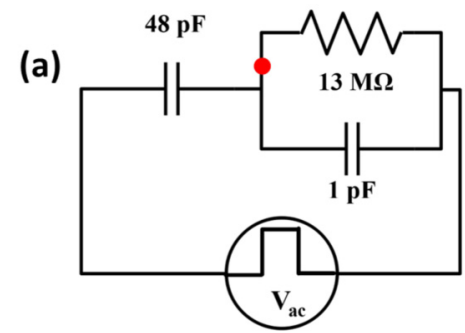

(b)

(c)
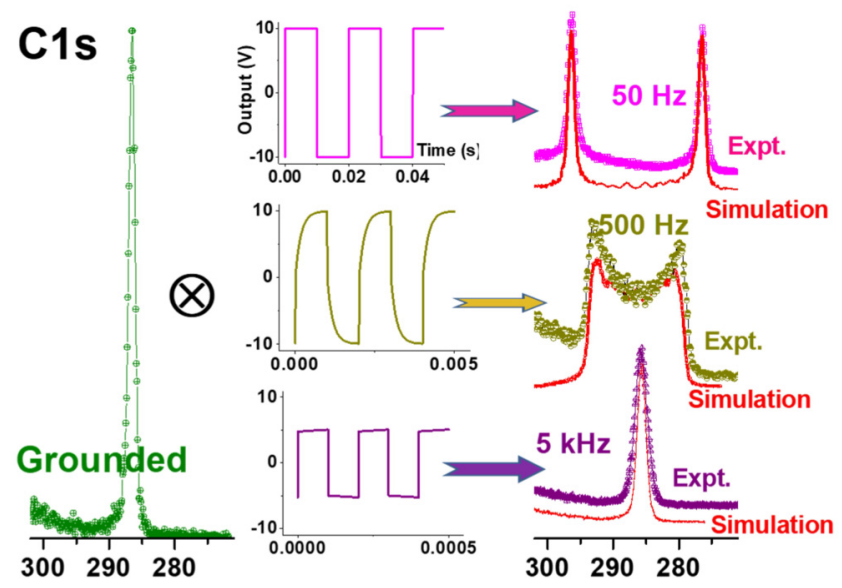

FIG. 20. $10 \mathrm{~V} S Q W A C$ actuation and its corresponding response simulated via SPICE. (a) Simplified equivalent circuit. (b) Unbiased C1s signal. (c) LT-Spice output waveforms. (d) Spectra obtained via the convolution of the unbiased signal with the corresponding waveforms at three different frequencies, together with the experimental measurements. All data refer to those recorded/simulated at the contact line (shown as the spot in the circuit). Adapted with permission from Uzundal et al., Langmuir 35, 16989 (2019). Copyright 2019, American Chemical Society.

behavior is encountered in ionic liquid interfaces and can be attributed to the enhanced electrical double-layer formation. ${ }^{84} \mathrm{~A}$ recent and detailed report showed that the fluoropolymer-water interfaces were spontaneously and negatively charged as a result of prolonged exposure to water and/or the repeated application of the EWOD process. ${ }^{85}$

In all of the previous works, this information had been derived using surface tension or streaming potential measurements, supplemented by electrical and/or electrochemical measurements and modeling, which all provide indirect evidence for the electrical potential developments due to charge accumulation. As has been advocated throughout this review, and will also be shown below, our methodology elucidates such potential developments directly.

Under DC bias applied to the liquid drop on this dielectric, no measurable current passes $(<10 \mathrm{pA})$, even under as large DC voltages as $180 \mathrm{~V}$. Hence, both C1s and O1s peaks of PEG display trivial potential dependent shifts of up to $180.0 \pm 0.1 \mathrm{eV}$ in their corresponding measured binding energies as shown explicitly in Fig. 21 . The estimated breakdown voltage is $180 \pm 20 \mathrm{~V}$. This value 


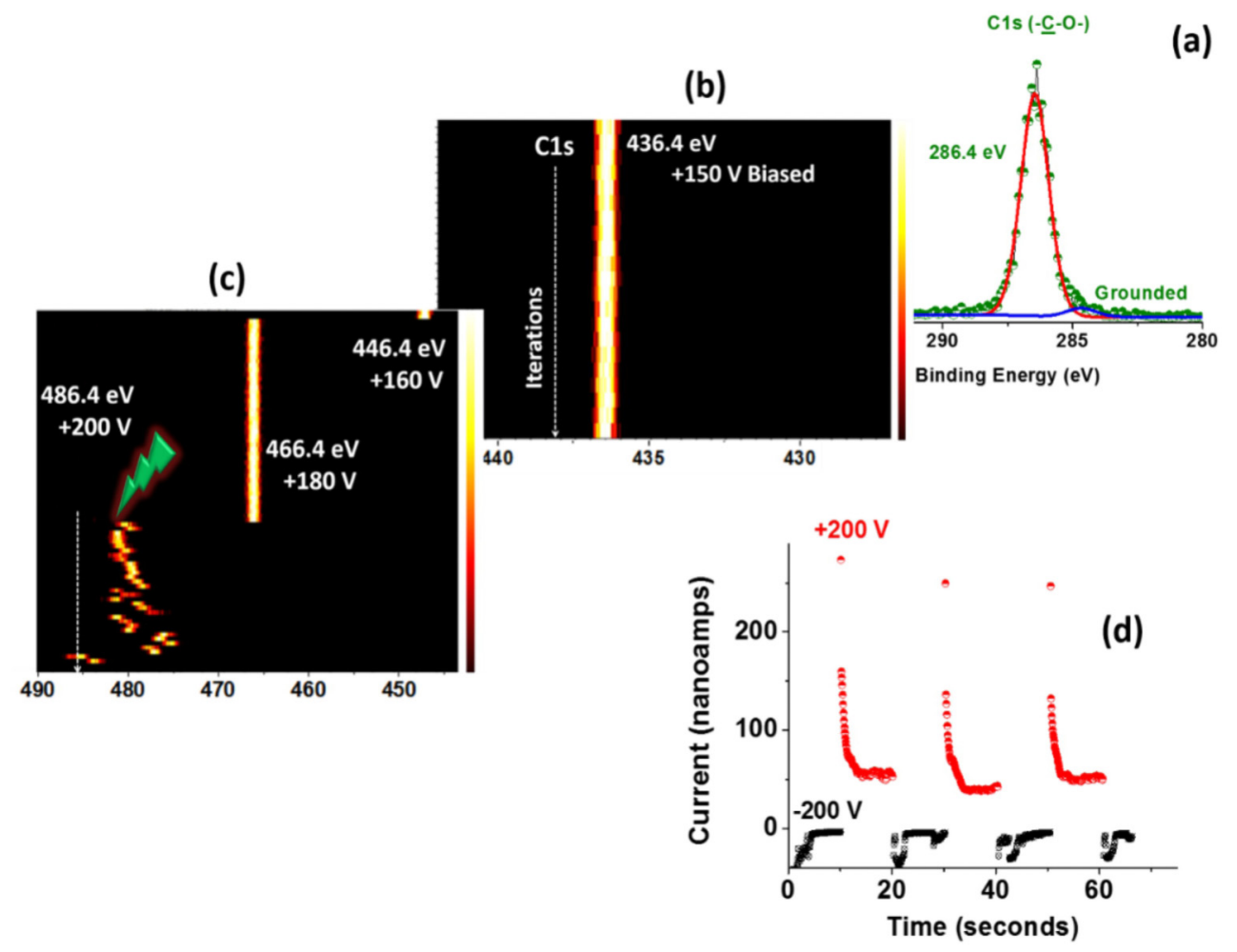

FIG. 21. C1s XP spectrum of pristine PEG drop recorded: (a) while the drop is grounded. (b) The same C1s region is recorded iteratively under $+150 \mathrm{~V}$ as a function of time, and (c) under $+160,+180$, and $+200 \mathrm{~V}$. Note that the drop undergoes several breakdown events under $+200 \mathrm{~V}$. (d) Recorded current after the breakdown events while the sample was subjected to several -200 and $+200 \mathrm{~V}$ pulses for with $10 \mathrm{~s}$ intervals. Adapted with permission from Uzundal et al., Langmuir 35, 16989 (2019). Copyright 2019, American Chemical Society.

is also consistent with our previously reported one on a similar PEG drop on CYTOP. ${ }^{38,39}$

However, exposing the liquid to voltages close to the breakdown threshold $<200 \mathrm{~V}$ results in measurable sporadic currents ranging from 0.05 to $2 \mu \mathrm{A}$. This current passage further causes a DC potential drop (IR drop), which translates to lower binding energy shifts in the spectra than the expected magnitude, see Fig. 21(c). Although after the first breakdown, the system displays several partial and even full recoveries, exposure to repeated breakdown brings the drop and the dielectric couple to a state with altered electrical properties, as can be seen from the asymmetric DC current measurements displayed in Fig. 21(d), recorded after the breakdown.

XPS data recorded under both DC and AC actuations are depicted in Fig. 22, which reveals that, in addition to the voltage asymmetry, the critical frequency is also lowered to $120 \mathrm{~Hz}$, such that the application of the $10 \mathrm{~V} \mathrm{SQW}$ actuation induces additional AC current passing through the system even at $50 \mathrm{~Hz}$. This can be attributed to an overall increase in the liquid's resistance (decrease in conductivity), since the RC is expected to also increase if one assumes that the capacitance of the dielectric is not altered, as formulated in Eq. (1) above.

After the breakdown, the asymmetric behavior of the system is further supported through the XPS data displayed in Fig. 22, better revealing the changes in the electrical properties. As such, under $+10 \mathrm{~V}$ DC bias, $\sim 56 \mathrm{nA}$ (measured) of leakage current passes through the system, causing a $-1.3 \mathrm{eV}$ shift (only $+8.7 \mathrm{eV}$ shift is measured instead of the trivial $+10.0 \mathrm{eV}$ ) in the binding energy (IR drop of $1.3 \mathrm{~V}$ ), after the breakdown. This simply implies that the rest of the voltage drops on the dielectric. These XPS measurements now allow us to assign $\left(1.3 \mathrm{~V} / 56 \times 10^{-9} \mathrm{~A}=\right) \sim 23$ and $\sim 150 \mathrm{M} \Omega$ resistance values to the liquid and the leaky dielectric, respectively, but only at the positive polarity. We emphasize that, although a small amount of current $(\sim 1 \mathrm{nA})$ is measured, no significant shifts are observed under $-10 \mathrm{~V}$ bias, which translates to a resistance value $(56 / 1 \times 155 \mathrm{M} \Omega=) \sim 9 \mathrm{G} \Omega$.

These two facts, (i) the polarity dependent (asymmetric) leakage behavior and (ii) the lower critical frequency measured after breakdown, cannot be explained by simple arguments, such as an increase in the resistance of the liquid or the formation of a simple leaky dielectric because the addition of a large leakage resistor would indeed cause less than $10.0 \mathrm{eV}$ shifts in the binding energy positions, but it should work symmetrically for both the positive and the negative cycles, contrary to the observed behavior. The overall system after the breakdown behaves like a diode. Therefore, we have also carried out simulations for the $\mathrm{C} 1 \mathrm{~s}$ spectra under DC and AC actuations. The results are shown in 
(a)

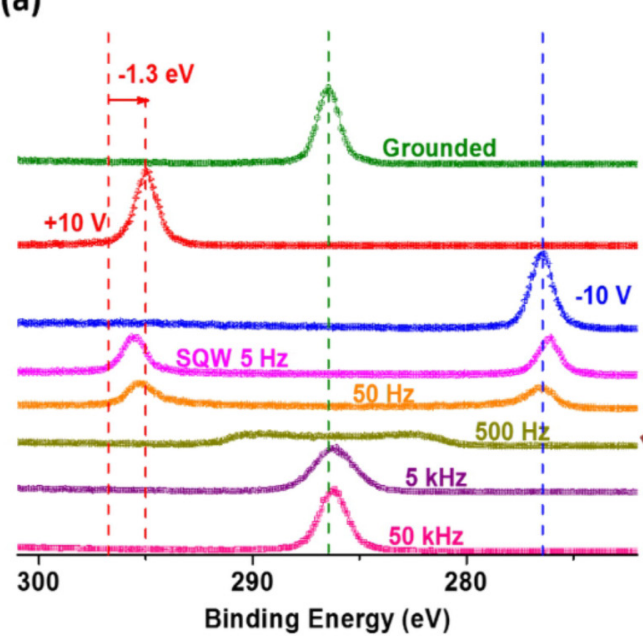

(c) Simulated

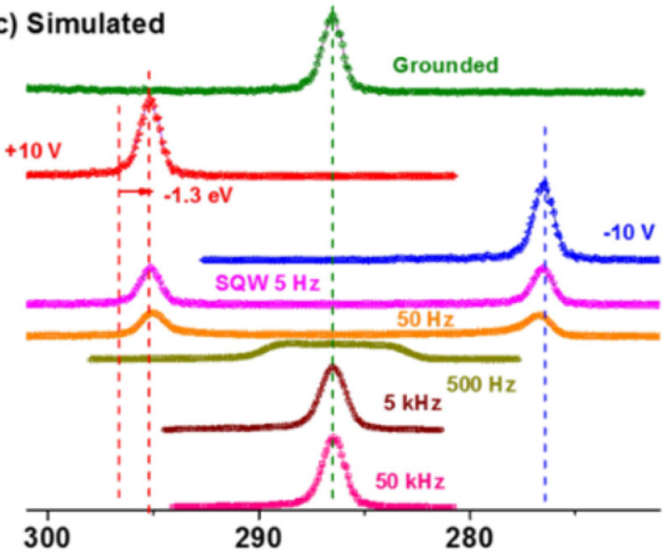

(b)

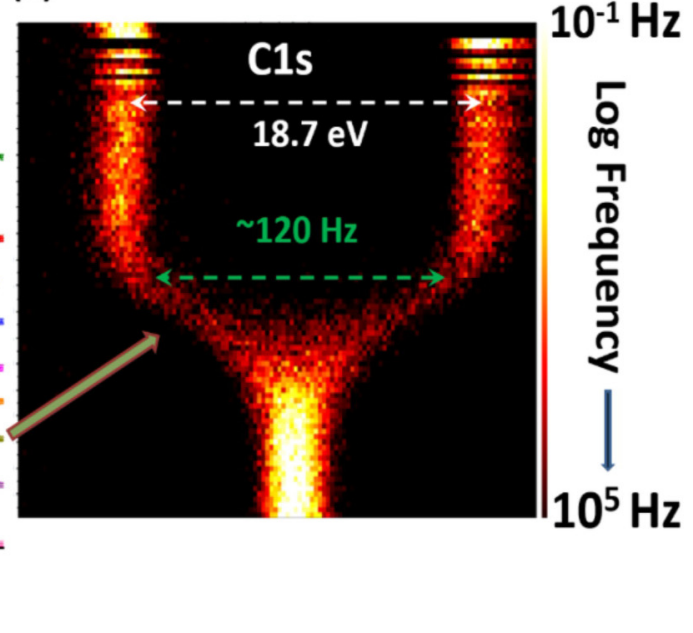

(d) Equivalent Circuit

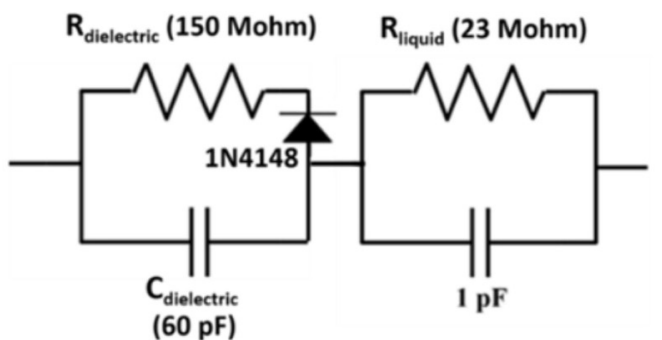

FIG. 22. (a) C1s region of the system after the breakdown, recorded under $0 \mathrm{~V}$ bias, and under +10 and $-10 \mathrm{~V} D C$ bias, together with $10 \mathrm{~V}$ SQW AC bias at four different frequencies. (b) The same data recorded using the snapshot mode with $1 \mathrm{~s}$ time resolution, while the drop is subjected to a logarithmic frequency sweep between $10^{-1}$ (top) to $10^{5} \mathrm{~Hz}$ (bottom). (c) Simulated XP spectra. (d) Equivalent circuit used for the simulation. Adapted with permission from Uzundal et al., Langmuir 35, 16989 (2019). Copyright 2019, American Chemical Society.

Fig. 22(c), together with the experimental data for the system after the breakdown.

The measured $\mathrm{C} 1 \mathrm{~s}$ spectra of the system after the breakdown in Fig. 22(a) and the simulated ones in Fig. 22(c) are surprisingly but may be fortuitously similar. If correct, our findings might suggest that a diode could represent either the electrical double layer at the PEG/Teflon interface or the leaky $\mathrm{SiO}_{2}-\mathrm{Si}$ underlayer, acting like an MOS capacitor. ${ }^{86}$

Incorporating simulation results yields two important new features, such as the abilities to (i) decouple the electrical properties of the liquid drop from those of the dielectric, and under diverse conditions; (ii) determine a decrease in the conductivity of the liquid after its breakdown, which is contrary to common understanding since strong electrical disturbances are usually expected to cause fragmentation and/or produce additional ionic moieties, hence increasing the conductivity; and (iii) surface a new type of asymmetric behavior of the postbreakdown system with respect to the polarity of the applied potential. This also points to an important question of whether all these obstacles, limiting the performance of electrowetting systems and devices, stem from the very same phenomenon, i.e., charge accumulation. The answer will undoubtedly pave the way to pursuing new strategies for circumventing them.

\section{SUMMARY AND CONCLUSIONS}

In summary, the highlighted applications of lab-based XPS in this review demonstrate the power of operando XPS, utilizing the application of electrical voltage bias in either the DC or AC forms, to investigate surfaces and interfaces of liquids with low volatility. We showed that in addition to its well-known capabilities such as 
chemical and elemental specificity as well as surface sensitivity, XPS is very powerful tool for probing local electrical potentials.

In the first part of the review, we showed exemplary cases on steady state information including time-dependent in situ reaction and ion intercalation monitoring under DC excitation. In the second part, we showed how AC excitation helps to reveal the dynamics of electrical potential developments by varying the frequency of the AC excitation to catch processes in action. Using the measured shifts in the apparent binding energy position representing the liquid, we extract local potentials in a totally noninvasive way, which are translated to electrical properties of the liquid and its interfaces.

One natural extension of the technique is to employ a combination of DC together with AC actuation, which is expected to be especially useful for improving the performance of the devices investigated.

In conclusion, our methodology introduces a new perspective for investigation of liquids and liquid/solid interface in vacuum. We also advocate that it will impact on several electrochemical issues, relevant especially for energy harvesting and storage. Last but not least, the simplicity of this approach makes it very adaptable for other experimental setups.

\section{ACKNOWLEDGMENTS}

This work was partially supported by the Scientific and Technological Research Council of Turkey (TUBITAK) through the Research Grant No. 118Z902, and is based on P.A.G.'s and M.T.C.'s Ph.D. theses. The authors would also like to extend their gratitude to several of their coauthors and colleagues, namely, Coskun Kocabas (presently in Manchester University, UK), Burak Ulgut, Yunus Emre Turkmen, Can-Berk Uzundal, and Ozgur Sahin, who have contributed significantly to development of our methodology throughout the last 5 years, and Frieder Mugele (University of Twente, The Netherlands) for his help in the electrowetting studies.

\section{REFERENCES}

${ }^{1}$ K. Siegbahn, ESCA, Atomic, Molecular and Solid State Structure Studied by Means of Electron Spectroscopy (Almqvist \& Wiksells, Uppsala, 1967).

${ }^{2}$ S. Södergren, H. Siegbahn, H. Rensmo, H. Lindström, A. Hagfeldt, and S.-E. Lindquist, J. Phys. Chem. B 101, 3087 (1997).

${ }^{3}$ Zhou, T. Baunach, V. Ivanova, D. M. Kolb, Langmuir 20, 4590 (2004).

${ }^{4}$ A. J. Pearse, E. Gillette, S. B. Lee, and G. W. Rubloff, Phys. Chem. Chem. Phys. 18, 19093 (2016).

${ }^{5}$ D. Tonti, C. Pettenkofer, and W. Jaegermann, Ionics 6, 196 (2000).

${ }^{6}$ A. Thißen, D. Ensling, M. Liberatore, Q. H. Wu, F. J. Fernandez Madrigal, M. S. Bhuvaneswari, R. Hunger, and W. Jaegermann, Ionics 15, 393 (2009).

${ }^{7}$ H. Sezen, A. A. Rockett, and S. Suzer, Anal. Chem. 84, 2990 (2012).

${ }^{8}$ H. Sezen and S. Suzer, Thin Solid Films 534, 1 (2013).

${ }^{\mathbf{9} E .}$ Yilmaz, H. Sezen, and S. Suzer, Angew. Chem. Int. Ed. 51, 5488 (2012).

${ }^{10}$ M. Copuroglu, P. Aydogan, E. O. Polat, C. Kocabas, and S. Süzer, Nano Lett. 14, 2837 (2014).

${ }^{11}$ P. Aydogan, O. Balci, C. Kocabas, and S. Suzer, Org. Electron. 37, 178 (2016).

${ }^{12}$ H. Siegbahn and K. Siegbahn, J. Electron Spectrosc. Relat. Phenom. 2, 319 (1973).

${ }^{13}$ S. Ghosal, J. C. Hemminger, H. Bluhm, B. S. Mun, E. L. D. Hebenstreit, G. Ketteler, D. F. Ogletree, F. G. Requejo, and M. Salmeron, Science 307, 563 (2005).
${ }^{14}$ F. Boekman, O. Bohman, and H. O. G. Siegbahn, J. Phys. Chem. 96, 2278 (1992).

${ }^{15}$ S. Axnanda et al., Sci. Rep. 5, 9788 (2015).

${ }^{16}$ M. Favaro, C. Valero-Vidal, J. Eichhorn, F. M. Toma, P. N. Ross, J. Yano, Z. Liu, and E. J. Crumlin, J. Mater. Chem. A 5, 11634 (2017).

${ }^{17}$ M. A. Brown, A. Beloqui Redondo, M. Sterrer, B. Winter, G. Pacchioni, Z. Abbas, and J. A. van Bokhoven, Nano Lett. 13, 5403 (2013).

${ }^{18}$ J. J. Velasco-Velez et al., Angew. Chem. Int. Ed. 54, 14554 (2015).

${ }^{19}$ H. Bluhm et al., J. Electron Spectrosc. Relat. Phenom. 150, 86 (2006).

${ }^{20}$ C. Zhang et al., ACS Catal. 2, 2297 (2012).

${ }^{21}$ M. F. Lichterman et al., Energy Environ. Sci. 8, 2409 (2015).

${ }^{22}$ A. Broderick, M. A. Rocha, Y. Khalifa, M. B. Shiflett, and J. T. Newberg, J. Phys. Chem. B 123, 2576 (2019).

${ }^{23}$ M. A. Brown, Z. Abbas, A. Kleibert, R. G. Green, A. Goel, S. May, and T. M. Squires, Phys. Rev. X 6, 011007 (2016).

${ }^{\mathbf{2 4}}$ M. Favaro, B. Jeong, P. N. Ross, J. Yano, Z. Hussain, Z. Liu, and E. J. Crumlin, Nat. Commun. 7, 12695 (2016).

${ }^{25}$ C. Arble, M. Jia, and J. T. Newberg, Surf. Sci. Rep. 73, 37 (2018).

${ }^{\mathbf{2 6}}$ K. R. J. Lovelock, I. J. Villar-Garcia, F. Maier, H.-P. Steinrück, and P. Licence, Chem. Rev. 110, 5158 (2010).

${ }^{27}$ F. Qiu, A. W. Taylor, S. Men, I. J. Villar-Garcia, and P. Licence, Phys. Chem. Chem. Phys. 12, 1982 (2010).

${ }^{28}$ A. Foelske-Schmitz, D. Weingarth, and R. Kötz, Electrochim. Acta 56, 10321 (2011).

${ }^{29}$ R. Wibowo, L. Aldous, R. M. J. Jacobs, N. S. A. Manan, and R. G. Compton, Chem. Phys. Lett. 509, 72 (2011).

${ }^{30}$ D. Weingarth, A. Foelske-Schmitz, A. Wokaun, and R. Kötz, Electrochem. Commun. 13, 619 (2011).

${ }^{31}$ T. Matsuda, N. Taccardi, J. Schwegler, P. Wasserscheid, H.-P. Steinrück, and F. Maier, ChemPhysChem 16, 1873 (2015).

${ }^{32}$ M. Olschewski, R. Gustus, O. Höfft, A. Lahiri, and F. Endres, J. Phys. Chem. C 121, 2675 (2017).

${ }^{33}$ M. T. Camci, P. Aydogan, B. Ulgut, C. Kocabas, and S. Suzer, Phys. Chem. Chem. Phys. 18, 28434 (2016).

${ }^{34}$ M. T. Camci, B. Ulgut, C. Kocabas, and S. Suzer, ACS Omega 2, 478 (2017).

${ }^{35}$ P. Aydogan Gokturk, U. Salzner, L. Nyulászi, B. Ulgut, C. Kocabas, and S. Suzer, Electrochim. Acta 234, 37 (2017).

${ }^{36}$ P. Aydogan Gokturk, S. E. Donmez, B. Ulgut, Y. E. Türkmen, and S. Suzer, New J. Chem. 41, 10299 (2017).

${ }^{37}$ P. Aydogan Gokturk, B. Ulgut, and S. Suzer, Langmuir 34, 7301 (2018).

${ }^{38}$ P. Aydogan Gokturk, B. Ulgut, and S. Suzer, Langmuir 35, 3319 (2019).

${ }^{39}$ C. B. Uzundal, P. Aydogan Gokturk, S. Suzer, and B. Ulgut, J. Phys. Chem. C 123, 13192 (2019).

${ }^{40}$ C. B. Uzundal, O. Sahin, P. Aydogan Gokturk, H. Wu, F. Mugele, B. Ulgut, and S. Suzer, Langmuir 35, 16989 (2019).

${ }^{41}$ M. Watanabe, M. L. Thomas, S. Zhang, K. Ueno, T. Yasuda, and K. Dokko, Chem. Rev. 117, 7190 (2017).

${ }^{42}$ A. J. Arduengo III, R. L. Harlow, and M. Kline, J. Am. Chem. Soc. 113, 361 (1991).

${ }^{43}$ M. N. Hopkinson, C. Richter, M. Schedler, and F. Glorius, Nature 510, 485 (2014).

${ }^{44}$ H. Wanzlick, Angew. Chem. Int. Ed. 1, 75 (1962).

${ }^{45}$ W. A. Herrmann, Angew. Chem. Int. Ed. 41, 1290 (2002).

${ }^{46}$ B. Gorodetsky, T. Ramnial, N. R. Branda, and J. A. Clyburne, Chem. Commun. 17, 1972 (2004).

${ }^{47}$ M. Feroci, I. Chiarotto, M. Orsini, R. Pelagalli, and A. Inesi, Chem. Commun. 48, 5361 (2012).

${ }^{48}$ E. F. Smith, F. J. Rutten, I. J. Villar-Garcia, D. Briggs, and P. Licence, Langmuir 22, 9386 (2006).

${ }^{49}$ I. J. Villar-Garcia, K. R. Lovelock, S. Men, and P. Licence, Chem. Sci. 5, 2573 (2014).

${ }^{50}$ S. Men, D. S. Mitchell, K. R. Lovelock, and P. Licence, ChemPhysChem 16, 2211 (2015).

${ }^{\mathbf{5 1}}$ K. R. Lovelock, E. F. Smith, A. Deyko, I. J. Villar-Garcia, P. Licence, and R. G. Jones, Chem. Commun. 46, 4866 (2007). 
${ }^{52}$ I. J. Villar-Garcia, E. F. Smith, A. W. Taylor, F. Qiu, K. R. Lovelock, R. G. Jones, and P. Licence, Phys. Chem. Chem. Phys. 13, 2797 (2011).

${ }^{53}$ M. Iida, C. Baba, M. Inoue, H. Yoshida, E. Taguchi, and H. Furusho, Chem. Eur. J. 14, 5047 (2008).

${ }^{54}$ A. Imanishi, M. Tamura, and S. Kuwabata, Chem. Commun. 13, 1775 (2009).

${ }^{55}$ T. Nakashima, Y. Hayakawa, M. Mori, and T. Kawai, Polym. J. 47, 171 (2015).

${ }^{56}$ J. Hu, Q. Yang, L. Yang, Z. Zhang, B. Su, Z. Bao, Q. Ren, H. Xing, and S. Dai, ACS Catal. 5, 6724 (2015).

${ }^{\mathbf{5 7}}$ K. Manojkumar, A. Sivaramakrishna, and K. Vijayakrishna, J. Nanopart. Res. 18, 103 (2016).

${ }^{58}$ M. A. Gelesky, A. P. Umpierre, G. Machado, R. R. Correia, W. C. Magno, J. Morais, G. Ebeling, and J. Dupont, J. Am. Chem. Soc. 127, 4588 (2005).

${ }^{\mathbf{5 9}}$ K.-I. Okazaki, T. Kiyama, K. Hirahara, N. Tanaka, S. Kuwabata, and T. Torimoto, Chem. Commun. 6, 691 (2008).

${ }^{60}$ Y. Hatakeyama, S. Takahashi, and K. Nishikawa, J. Phys. Chem. C 114, 11098 (2010).

${ }^{61}$ Y. Gao, A. Voigt, M. Zhou, and K. Sundmacher, Eur. J. Inorg. Chem. 2008, 3769 (2008).

${ }^{62}$ M. T. Camci, B. Ulgut, C. Kocabas, and S. Suzer, J. Phys. Chem. C 122, 11883 (2018).

${ }^{63}$ H. Cohen, J. Electron Spectrosc. Relat. Phenom. 176, 24 (2010).

${ }^{64}$ E. O. Polat, O. Balc1, and C. Kocabas, Sci. Rep. 4, 6484 (2014).

${ }^{65}$ M. G. Pollack, R. B. Fair, and A. D. Shenderov, Appl. Phys. Lett. 77, 1725 (2000).

${ }^{66}$ B. Berge and J. Peseux, Eur. Phys. J. E 3, 159 (2000).

${ }^{67}$ S. Kuiper and B. Hendriks, Appl. Phys. Lett. 85, 1128 (2004).

${ }^{68}$ V. Srinivasan, V. K. Pamula, and R. B. Fair, Lab Chip 4, 310 (2004).

${ }^{69}$ H. Ren, R. B. Fair, and M. G. Pollack, Sens. Actuators B Chem. 98, 319 (2004).
${ }^{70}$ D. Chatterjee, B. Hetayothin, A. R. Wheeler, D. J. King, and R. L. Garrell, Lab Chip 6, 199 (2006).

71. Gong, Lab Chip 8, 898 (2008).

${ }^{72}$ J. Hong, Y. K. Kim, D.-J. Won, J. Kim, and S. J. Lee, Sci. Rep. 5, 10685 (2015).

${ }^{73}$ S. Sadeghi, H. Ding, G. J. Shah, S. Chen, P. Y. Keng, C.-J. C. Kim, and R. M. van Dam, Anal. Chem. 84, 1915 (2012).

${ }^{74}$ F. Mugele and J.-C. Baret, J. Phys. Condens. Matter 17, R705 (2005).

${ }^{75} \mathrm{~F}$. Mugele and J. Heikenfeld, Electrowetting: Fundamental Principles and Practical Applications (Wiley, New York, 2018).

${ }^{76}$ X. Zeng, K. Zhang, G. Tao, Z. Zeng, S.-K. Fan, and J. Zhou, Jpn. J. Appl. Phys. 53, 060304 (2014).

${ }^{77} \mathrm{~K}$. Zhang, L. Chao, and J. Zhou, Materials 11, 1332 (2018).

${ }^{78}$ G. M. Panhwar, R. Mysyk, T. F. Rojo, S. Shaikhutdinov, and O. Bondarchuk, Langmuir 34, 14528 (2018).

${ }^{79}$ J. S. Hong, S. H. Ko, K. H. Kang, and I. S. Kang, Microfluid. Nanofluid. 5, 263 (2008).

${ }^{80}$ See https://www.analog.com/en/design-center/design-tools-andcalculators/LTspice-s.

${ }^{81}$ W. K. Sikkema, A. B. Metzger, T. Wang, and J. M. Tour, Surg. Neurol. Int. 8, 84 (2017).

${ }^{82} \mathrm{H}$. Verheijen and M. Prins, Langmuir 15, 6616 (1999).

${ }^{83}$ A. Quinn, R. Sedev, and J. Ralston, J. Phys. Chem. B 107, 1163 (2003).

${ }^{84}$ S. Millefiorini, A. H. Tkaczyk, R. Sedev, J. Efthimiadis, and J. Ralston, J. Am. Chem. Soc. 128, 3098 (2006).

${ }^{85}$ A. G. Banpurkar, Y. Sawane, S. M. Wadhai, C. U. Murade, I. Siretanu, D. van den Ende, and F. Mugele, Faraday Discuss. 199, 29 (2017).

${ }^{86}$ S. Rangan, M. Kalyanikar, J. Duan, G. Liu, R. A. Bartynski, E. Y. Andrei, L. Feldman, and E. Garfunkel, J. Phys. Chem. Lett. 7, 3434 (2016). 